\title{
SYNTHESIS AND ANTIBACTERIAL EVALUATION OF NOVEL 3,6- DISUBSTITUTED COUMARIN DERIVATIVES
}

${ }^{1}$ Ravibabu Velpula, ${ }^{1}$ Ramesh Gondru, ${ }^{2}$ Yashodhara Velivela and ${ }^{1}$ Rajitha Bavantula* ${ }^{I}$ Department of Chemistry, National Institute of Technology, Warangal-506004, A.P., India

${ }^{2}$ Department of Biotechnology, Kakatiya University, Warangal-506009, Telangana, India

\section{EXPERIMENTAL SECTION}

\section{Materials and instrumentation.}

All the reagents and solvents were purchased from Aldrich/Merck and used without further purifications. Melting points were determined in open capillaries using Stuart SMP30 apparatus and are uncorrected. The progress of the reactions as well as purity of compounds was monitored by thin layer chromatography with $F_{254}$ silica-gel precoated sheets using hexane/ethyl acetate 8/2 as eluent; UV light and iodine vapours were used for detection. IR spectra were recorded on a Perkin-Elmer 100S spectrometer utilizing KBr pellets. ${ }^{1} \mathrm{H}$ NMR and ${ }^{13} \mathrm{C}$ NMR spectra were obtained at $400 \mathrm{MHz}$ and $100 \mathrm{MHz}$ respectively on Bruker using DMSO- $d_{6}$ as solvent and TMS as internal standard. Elemental analyses were performed on a Carlo-Erba model EA1108 analytical unit. Mass spectra were recorded on a Jeol JMSD-300 spectrometer.

General procedure for the synthesis of ethyl-2-(3-acetyl-2-oxo-2H-chromen-6-yl)-4methylthiazole-5-carboxylate (3)

A mixture of ethyl 2-(3-formyl-4-hydroxyphenyl)-4-methylthiazole-5-carboxylate (1) (0.1 mol) and ethylacetoacetate $(2)(0.12 \mathrm{~mol})$ were dissolved in ethanol and cooled at $0-5{ }^{0} \mathrm{C}$. Piperidine $(1 \mathrm{~mL})$ was added drop wise to this mixture while stirring. The reaction mixture left overnight, resulting in the formation of yellow colored solid. Purification by recrystallization from ethanol to afford analytically pure product.

General procedure for the synthesis of 3,6-disubstituted coumarin derivatives (6a-l)

A mixture of ethyl-2-(3-acetyl-2-oxo-2H-chromen-6-yl)-4-methylthiazole-5-carboxylate (3, 1 $\mathrm{mmol})$, thiosemicarbazide $(1 \mathrm{mmol})$ and various phenacyl bromides (5a-g) / 3-(2-bromoacetyl)$2 H$-chromen-2-ones (5h-k) / 2-(2-bromoacetyl)-3H-benzo[f]chromen-3-one (5l) (1 mmol) were dissolved in $10 \mathrm{~mL}$ of absolute ethanol in the presence of catalytic amount of acetic acid and heated at refluxing temperature for 2-4 hrs. Monitored the reaction by TLC and poured contents in an ice cold water, the solid obtained was filtered, dried and recrystallised in ethanol. 


\section{Spectral data of synthesized compounds $(3,6 a-61)$}

\section{Ethyl 2-(3-acetyl-2-oxo-2H-chromen-6-yl)-4-methylthiazole-5-carboxylate (3)}

Yellow solid; mp. 194-196 ${ }^{\circ} \mathrm{C}$; ${ }^{1} \mathrm{H}$ NMR (400MHz, DMSO- $\left.d_{6}\right)$ : 1.32 (t, $3 \mathrm{H}, J=7.2 \mathrm{~Hz}$ ), 2.59 (s, 3H), 2.71 (s, 3H), 4.29-4.34 (m, 2H), 7.58 (d, 1H, $J=8.8 \mathrm{~Hz}), 8.29-8.32(\mathrm{~m}, 1 \mathrm{H}), 8.63$ (s, $1 \mathrm{H}), 8.75(\mathrm{~s}, 1 \mathrm{H}) ;{ }^{13} \mathrm{C}$ NMR $\left(100 \mathrm{MHz}, \mathrm{DMSO}-d_{6}\right): \delta 14.0,17.1,29.9,61.2,117.2,118.7$, 125.4, 128.7, 131.8, 146.3, 149.4, 156.2, 156.7, 157.9, 160.4, 161.2, 195.0;

\section{Ethyl-4-methyl-2-(2-0xo-3-(1-(2-(4-phenylthiazol-2-yl)hydrazono)ethyl)-2H-chromen-6-} yl)thiazole-5-carboxylate (6a)

Pale yellow solid; mp. 224-226 ${ }^{\circ} \mathrm{C}$; IR $\left(\mathrm{KBr}, \mathrm{cm}^{-1}\right) v_{\max }$ : $3404(\mathrm{NH}), 1741(\mathrm{C}=\mathrm{O}), 1718(\mathrm{C}=\mathrm{O})$, $1611(\mathrm{C}=\mathrm{N}), 1565(\mathrm{C}=\mathrm{C}) ;{ }^{1} \mathrm{H}$ NMR $\left(400 \mathrm{MHz}, \mathrm{DMSO}-d_{6}\right): \delta 1.31(\mathrm{t}, 3 \mathrm{H}, J=7.2 \mathrm{~Hz}), 2.29(\mathrm{~s}$, $3 \mathrm{H}), 2.71(\mathrm{~s}, 3 \mathrm{H}), 4.28-4.33(\mathrm{~m}, 2 \mathrm{H}), 7.29-7.44(\mathrm{~m}, 2 \mathrm{H}), 7.55(\mathrm{~d}, 1 \mathrm{H}, J=8.8 \mathrm{~Hz}), 7.89(\mathrm{~d}, 2 \mathrm{H}, J$ $=7.2 \mathrm{~Hz}), 8.19-8.22(\mathrm{~m}, 1 \mathrm{H}), 8.30(\mathrm{~s}, 1 \mathrm{H}), 8.56-8.62(\mathrm{~m}, 2 \mathrm{H}), 8.74(\mathrm{~s}, 1 \mathrm{H}), 11.45(\mathrm{~s}, 1 \mathrm{H}) ;{ }^{13} \mathrm{C}$ NMR $\left(100 \mathrm{MHz}, \mathrm{DMSO}-d_{6}\right): \delta 14.0,16.0,17.1,61.2,104.4,116.9,119.5,121.5,125.5,127.2$, 127.4, 128.5, 129.7, 134.5, 139.9, 144.4, 154.7, 158.5, 160.2, 161.2, 167.3, 169.2; MS (ESI) $m / z$ : $531[\mathrm{M}+1]^{+}$; Anal. Calcd. for $\mathrm{C}_{27} \mathrm{H}_{22} \mathrm{~N}_{4} \mathrm{O}_{4} \mathrm{~S}_{2}$ : C, 61.12; H, 4.18; N, 10.56. Found: C, 61.24; H, $4.06 ; \mathrm{N}, 10.61$.

Ethyl-2-(3-(1-(2-(4-(4-chlorophenyl)thiazol-2-yl)hydrazono)ethyl)-2-oxo-2H-chromen-6-yl)4-methylthiazole-5-carboxylate (6b)

Yellow solid; mp. 188-190 ${ }^{\circ} \mathrm{C}$; IR $\left(\mathrm{KBr}, \mathrm{cm}^{-1}\right)$ v $_{\max }: 3404(\mathrm{NH}), 1742(\mathrm{C}=\mathrm{O}), 1718(\mathrm{C}=\mathrm{O}), 1611$ $(\mathrm{C}=\mathrm{N}) 1565(\mathrm{C}=\mathrm{C}), 759(\mathrm{C}-\mathrm{Cl}) ;{ }^{1} \mathrm{H}$ NMR $\left(400 \mathrm{MHz}, \mathrm{DMSO}-d_{6}\right): \delta 1.31(\mathrm{t}, 3 \mathrm{H}, J=7.2 \mathrm{~Hz}), 2.59$ (s, 3H), 2.70 (s, 3H), 4.28-4.34 (m, 2H), 7.45-7.59 (m, 2H), 7.90 (d, 1H, J=8.4 Hz), 8.20 (d, 1H, $8.8 \mathrm{~Hz}), 8.29$ (t, $2 \mathrm{H}, J=7.2 \mathrm{~Hz}), 8.55(\mathrm{~s}, 1 \mathrm{H}), 8.62(\mathrm{~s}, 1 \mathrm{H}), 8.74(\mathrm{~s}, 1 \mathrm{H}), 11.47(\mathrm{~s}, 1 \mathrm{H}) ;{ }^{13} \mathrm{C}$ NMR (100 MHz, DMSO- $\left.d_{6}\right): \delta 14.0,17.1,29.9,61.2,117.2,118.7,121.5,121.7,125.3,127.2,128.5$, 128.7, 131.8, 146.3, 156.0, 157.8, 160.2, 161.2, 194.8; MS (ESI) m/z: 567 [M+2]+; Anal. Calcd. for $\mathrm{C}_{27} \mathrm{H}_{21} \mathrm{ClN}_{4} \mathrm{O}_{4} \mathrm{~S}_{2}$ : C, 57.39; H, 3.75; N, 9.92. Found: C, 57.47; H, 3.62; N, 9.83.

Ethyl-2-(3-(1-(2-(4-(4-bromophenyl)thiazol-2-yl)hydrazono)ethyl)-2-oxo-2H-chromen-6-yl)4-methylthiazole-5-carboxylate (6c)

Yellow solid; mp. 239-241 ${ }^{\circ} \mathrm{C}$; IR $\left(\mathrm{KBr}, \mathrm{cm}^{-1}\right) v_{\max }: 3415(\mathrm{NH}), 1718(\mathrm{C}=\mathrm{O}), 1610(\mathrm{C}=\mathrm{N}), 1569$ $(\mathrm{C}=\mathrm{C}), 718(\mathrm{C}-\mathrm{Br}) ;{ }^{1} \mathrm{H}$ NMR $\left(400 \mathrm{MHz}, \mathrm{DMSO}-d_{6}\right): \delta 1.31(\mathrm{t}, 3 \mathrm{H}, J=7.2 \mathrm{~Hz}), 2.28(\mathrm{~s}, 3 \mathrm{H}), 2.71$ (s, 3H), 4.28-4.33 (m, 2H), 7.46 (s, 1H), 7.54 (d, 1H, $J=8.4 \mathrm{~Hz}), 7.61$ (d, 2H, $J=8.8 \mathrm{~Hz}), 7.83$ $(\mathrm{d}, 2 \mathrm{H}, J=8.4 \mathrm{~Hz}), 8.19-8.22(\mathrm{~m}, 1 \mathrm{H}), 8.29(\mathrm{~s}, 1 \mathrm{H}), 8.56(\mathrm{~s}, 1 \mathrm{H}), 11.44(\mathrm{~s}, 1 \mathrm{H}),{ }^{13} \mathrm{C}$ NMR $(100$ 
MHz, DMSO- $\left.d_{6}\right): \delta 14.0,16.0,17.1,61.2,116.9,121.3,126.6,127.3,127.5,128.5,129.7,131.2$, $131.4,131.8,132.6,140.2,154.7,158.4,160.2,161.2,167.3,168.2$; MS (ESI) $\mathrm{m} / \mathrm{z}: 610$ [M+1] $]^{+}$; Anal. Calcd. for $\mathrm{C}_{27} \mathrm{H}_{21} \mathrm{BrN}_{4} \mathrm{O}_{4} \mathrm{~S}_{2}$ : C, 53.20; H, 3.47; N, 9.19. Found: C, 53.41; H, 3.33; N, 9.24.

Ethyl-4-methyl-2-(3-(1-(2-(4-(4-nitrophenyl)thiazol-2-yl)hydrazono)ethyl)-2-oxo-2Hchromen-6-yl)thiazole-5-carboxylate (6d)

Yellow solid; mp. 196-198 ${ }^{\circ} \mathrm{C}$; IR (KBr, cm $\left.{ }^{-1}\right) v_{\max }: 3416(\mathrm{NH}), 1735(\mathrm{C}=\mathrm{O}), 1598(\mathrm{C}=\mathrm{N}), 1340$ $\left(\mathrm{NO}_{2}\right) ;{ }^{1} \mathrm{H}$ NMR (400MHz, DMSO-d $\left.)_{6}\right): \delta 1.32$ (t, 3H, $\left.J=7.2 \mathrm{~Hz}\right), 2.30(\mathrm{~s}, 3 \mathrm{H}), 2.72(\mathrm{~s}, 3 \mathrm{H})$, 4.29-4.34 m, 2H), 7.56-7.61 (m, 1H), 7.79 (s, 1H), 8.15 (d, 1H, J=8.8 Hz), 8.22-8.33 (m, 3H), $8.59(\mathrm{~s}, 1 \mathrm{H}), 8.66(\mathrm{~s}, 1 \mathrm{H}), 8.76(\mathrm{~s}, 1 \mathrm{H}), 11.60(\mathrm{~s}, 1 \mathrm{H}) ;{ }^{13} \mathrm{C}$ NMR $\left(100 \mathrm{MHz}, \mathrm{DMSO}-d_{6}\right): \delta 14.1$, $17.0,61.2$, 117.2, 118.1, 121.4, 123.0, 125.3, 127.2, 128.2, 129.5, 132.2, 146.1, 156.0, 158.8, 160.2, 162.3; MS (ESI) m/z: $576[\mathrm{M}+1]^{+}$; Anal. Calcd. for $\mathrm{C}_{27} \mathrm{H}_{21} \mathrm{~N}_{5} \mathrm{O}_{6} \mathrm{~S}_{2}: \mathrm{C}, 56.34 ; \mathrm{H}, 3.68$; N, 12.17. Found: C, 56.44; H, 3.53; N, 12.23.

Ethyl-4-methyl-2-(2-oxo-3-(1-(2-(4-(p-tolyl)thiazol-2-yl)hydrazono)ethyl)-2H-chromen-6yl)thiazole-5-carboxylate (6e)

Yellow solid; mp. $235-237{ }^{\circ} \mathrm{C}$; IR ( $\left.\mathrm{KBr}, \mathrm{cm}^{-1}\right) v_{\max }: 3412(\mathrm{NH}), 1718(\mathrm{C}=\mathrm{O}), 1622(\mathrm{C}=\mathrm{N}), 1578$ $(\mathrm{C}=\mathrm{C}) ;{ }^{1} \mathrm{H}$ NMR $\left(400 \mathrm{MHz}, \mathrm{DMSO}-d_{6}\right): \delta 1.31(\mathrm{t}, 3 \mathrm{H}, J=7.2 \mathrm{~Hz}), 2.28(\mathrm{~s}, 3 \mathrm{H}), 2.32(\mathrm{~s}, 3 \mathrm{H})$, $2.71(\mathrm{~s}, 3 \mathrm{H}), 4.28-4.33(\mathrm{~m}, 2 \mathrm{H}), 7.22(\mathrm{~d}, 2 \mathrm{H}, J=8.0 \mathrm{~Hz}), 7.29(\mathrm{~s}, 1 \mathrm{H}), 7.54(\mathrm{~d}, 1 \mathrm{H}, J=8.8 \mathrm{~Hz})$, $7.77(\mathrm{~d}, 2 \mathrm{H}, J=8.0 \mathrm{~Hz}), 8.19-8.22(\mathrm{~m}, 1 \mathrm{H}), 8.29(\mathrm{~s}, 1 \mathrm{H}), 8.56(\mathrm{~s}, 1 \mathrm{H}), 11.45(\mathrm{~s}, 1 \mathrm{H}) ;{ }^{13} \mathrm{C}$ NMR (100 MHz, DMSO-d $\left.d_{6}\right): \delta 14.0,16.0,17.1,20.7,61.1,103.4,116.9,119.5,121.5,125.4,127.1$, $128.4,129.1,129.6,131.8,136.7,139.9,144.3,154.7,158.5,160.20,161.2,167.3,169.1$; MS (ESI) $m / z: 545[\mathrm{M}+1]^{+}$; Anal. Calcd. for $\mathrm{C}_{28} \mathrm{H}_{24} \mathrm{~N}_{4} \mathrm{O}_{4} \mathrm{~S}_{2}$ : C, 61.75; H, 4.44; N, 10.29. Found: C, 61.87; H, 4.21; N, 10.12.

Ethyl-2-(3-(1-(2-(4-(4-methoxyphenyl)thiazol-2-yl)hydrazono)ethyl)-2-oxo-2H-chromen-6yl)-4-methylthiazole-5-carboxylate (6f)

Red solid; mp. 216-218 ${ }^{\circ} \mathrm{C}$; IR (KBr, cm $\left.{ }^{-1}\right) v_{\text {max }}: 3418(\mathrm{NH}), 1713(\mathrm{C}=\mathrm{O}), 1612(\mathrm{C}=\mathrm{N}), 1186(\mathrm{C}-$ O-C); ${ }^{1} \mathrm{H}$ NMR (400MHz, DMSO-d $): \delta 1.33$ (t, 3H, J=7.2 Hz), 2.31 (s, 3H), 2.73 (s, 3H), 3.69 (s, 3H), 4.30-4.36 (m, 2H), $7.30(\mathrm{~d}, 2 \mathrm{H}, J=7.6 \mathrm{~Hz}), 7.57$ (d, 1H, J=8.4 Hz), $7.92(\mathrm{~d}, 1 \mathrm{H}, J=$ $7.2 \mathrm{~Hz}), 8.20-8.24(\mathrm{~m}, 1 \mathrm{H}), 8.33(\mathrm{~s}, 1 \mathrm{H}), 8.55-8.60(\mathrm{~m}, 2 \mathrm{H}), 8.76(\mathrm{~s}, 1 \mathrm{H}), 11.39(\mathrm{~s}, 1 \mathrm{H}) ;{ }^{13} \mathrm{C}$ NMR (100 MHz, DMSO- $\left.d_{6}\right): \delta 14.6,16.2,18.0,55.0,61.5,104.6,117.1,119.0,121.5,125.9$, $127.3,128.5,129.2,129.9,132.3,136.8,139.9,144.3,155.9,158.6,160.3,162.5,168.3,169.4$; 
MS (ESI) $m / z: 561[\mathrm{M}+1]^{+}$; Anal. Calcd. for $\mathrm{C}_{28} \mathrm{H}_{24} \mathrm{~N}_{4} \mathrm{O}_{5} \mathrm{~S}_{2}: \mathrm{C}, 59.98 ; \mathrm{H}, 4.31 ; \mathrm{N}, 9.99$. Found: C, 60.14; H, 4.19; N, 10.13.

Ethyl-2-(3-(1-(2-(4-([1,1'-biphenyl]-4-yl)thiazol-2-yl)hydrazono)ethyl)-2-oxo-2H-chromen6-yl)-4-methylthiazole-5-carboxylate (6g)

Pale yellow solid; mp. 209-211 ${ }^{\circ} \mathrm{C}$; IR ( $\left.\mathrm{KBr}, \mathrm{cm}^{-1}\right) v_{\max }: 3427(\mathrm{NH}), 1728(\mathrm{C}=\mathrm{O}), 1613(\mathrm{C}=\mathrm{N})$, $1577(\mathrm{C}=\mathrm{C}) ;{ }^{1} \mathrm{H}$ NMR (400MHz, DMSO-d $): \delta 1.32$ (t, 3H, $\left.J=6.8 \mathrm{~Hz}\right), 2.30(\mathrm{~s}, 3 \mathrm{H}), 2.71(\mathrm{~s}$, $3 \mathrm{H}), 4.28-4.34(\mathrm{~m}, 2 \mathrm{H}), 7.37(\mathrm{t}, 1 \mathrm{H}, J=7.2 \mathrm{~Hz}), 7.45-7.50(\mathrm{~m}, 3 \mathrm{H}), 7.55(\mathrm{~d}, 1 \mathrm{H}, J=8.8 \mathrm{~Hz})$, 7.71-7.74 (m, 4H), $7.98(\mathrm{~d}, 2 \mathrm{H}, J=8.4 \mathrm{~Hz}), 8.20-8.22(\mathrm{~m}, 1 \mathrm{H}), 8.30(\mathrm{~s}, 1 \mathrm{H}), 8.56(\mathrm{~s}, 1 \mathrm{H}), 11.48$ (s, 1H); MS (ESI) m/z: $606[\mathrm{M}]^{+}$; Anal. Calcd. for $\mathrm{C}_{33} \mathrm{H}_{26} \mathrm{~N}_{4} \mathrm{O}_{4} \mathrm{~S}_{2}: \mathrm{C}, 65.33 ; \mathrm{H}, 4.32 ; \mathrm{N}, 9.23$. Found: C, 65.51; H, 4.24; N, 9.14.

Ethyl-4-methyl-2-(2-oxo-3-(1-(2-(4-(2-oxo-2H-chromen-3-yl)thiazol-2-yl)hydrazono)ethyl)2H-chromen-6-yl)thiazole-5-carboxylate (6h)

Yellow solid; mp. 220-222 ${ }^{\circ} \mathrm{C}$; IR ( $\left.\mathrm{KBr}, \mathrm{cm}^{-1}\right) v_{\max }: 3416(\mathrm{NH}), 1717(\mathrm{C}=\mathrm{O}), 1608(\mathrm{C}=\mathrm{N}), 1563$ $(\mathrm{C}=\mathrm{C}) ;{ }^{1} \mathrm{H}$ NMR $\left(400 \mathrm{MHz}, \mathrm{DMSO}-d_{6}\right): \delta 1.32(\mathrm{t}, 3 \mathrm{H}, J=7.2 \mathrm{~Hz}), 2.30(\mathrm{~s}, 3 \mathrm{H}), 2.71(\mathrm{~s}, 3 \mathrm{H})$, 4.29-4.34 (m, 2H), $7.41(\mathrm{~d}, 1 \mathrm{H}, J=8.0 \mathrm{~Hz}), 7.46(\mathrm{~d}, 1 \mathrm{H}, J=8.0 \mathrm{~Hz}), 7.56(\mathrm{~d}, 1 \mathrm{H}, J=9.2 \mathrm{~Hz})$, $7.63(\mathrm{~d}, 1 \mathrm{H}, J=7.2 \mathrm{~Hz}), 7.82(\mathrm{t}, 2 \mathrm{H}, J=7.2 \mathrm{~Hz}), 8.21-8.23(\mathrm{~m}, 2 \mathrm{H}), 8.32(\mathrm{~s}, 1 \mathrm{H}), 8.59$ (s, 1H), $11.52(\mathrm{~s}, 1 \mathrm{H}) ;{ }^{13} \mathrm{C} \mathrm{NMR}\left(100 \mathrm{MHz}, \mathrm{DMSO}-d_{6}\right): \delta$ 14.4, 17.2, 19.0, 104.4, 116.9, 120.1, 120.9, 123.9 , 124.2, 127.2, 128.6, 128.9, 132.0, 133.1, 138.6, 148.7, 158.6, 160.2, 161.4, 167.4, 167.9, 168.8; MS (ESI) m/z: $598[\mathrm{M}]^{+}$; Anal. Calcd. for $\mathrm{C}_{30} \mathrm{H}_{22} \mathrm{~N}_{4} \mathrm{O}_{6} \mathrm{~S}_{2}$ : C, 60.19; H, 3.70; N, 9.36. Found: C, 60.32; H, 3.86; N, 9.19.

Ethyl-2-(3-(1-(2-(4-(6-chloro-2-oxo-2H-chromen-3-yl)thiazol-2-yl)hydrazono)ethyl)-2-oxo2H-chromen-6-yl)-4-methylthiazole-5-carboxylate (6i)

Shiny bright yellow; mp. 215-217 ${ }^{\circ} \mathrm{C}$; IR $\left(\mathrm{KBr}, \mathrm{cm}^{-1}\right) \mathrm{v}_{\max }: 3417(\mathrm{NH}), 1736(\mathrm{C}=\mathrm{O}), 1718$ $(\mathrm{C}=\mathrm{O}), 1619(\mathrm{C}=\mathrm{N}), 1565(\mathrm{C}=\mathrm{C}), 757(\mathrm{C}-\mathrm{Cl}) ;{ }^{1} \mathrm{H}$ NMR $\left(400 \mathrm{MHz}, \mathrm{DMSO}-d_{6}\right): \delta 1.32(\mathrm{t}, 3 \mathrm{H}, J=$ 7.6), 2.30 (s, 3H), 2.71 (s, 3H), 4.29-4.34 (m, 2H), 7.50 (d, 1H, J=8.8 Hz), 7.54-7.67 (m, 1H), $7.85(\mathrm{~s}, 1 \mathrm{H}), 7.97(\mathrm{~d}, 1 \mathrm{H}, J=7.2 \mathrm{~Hz}), 8.22(\mathrm{~d}, 1 \mathrm{H}, J=7.2 \mathrm{~Hz}), 8.31$ (t, 1H, $J=6.4 \mathrm{~Hz}), 8.51$ (s, $1 \mathrm{H}), 8.58(\mathrm{~s}, 1 \mathrm{H}), 8.65(\mathrm{~d}, 1 \mathrm{H}, J=6.0 \mathrm{~Hz}), 8.76(\mathrm{~s}, 1 \mathrm{H}), 11.55$ (s, 1H); MS (ESI) $m / z: 634$ $[\mathrm{M}+1]^{+}$; Anal. Calcd. for $\mathrm{C}_{30} \mathrm{H}_{21} \mathrm{ClN}_{4} \mathrm{O}_{6} \mathrm{~S}_{2}:$ C, 56.91; H, 3.34; N, 8.85. Found: C, 56.84; H, 3.48; N, 8.62.

Ethyl-2-(3-(1-(2-(4-(6-bromo-2-oxo-2H-chromen-3-yl)thiazol-2-yl)hydrazono)ethyl)-2-oxo2H-chromen-6-yl)-4-methylthiazole-5-carboxylate (6j) 
Yellow solid; mp. 228-230 ${ }^{\circ} \mathrm{C}$; IR ( $\left.\mathrm{KBr}, \mathrm{cm}^{-1}\right)$ v $\max _{2} 3419(\mathrm{NH}), 1735(\mathrm{C}=\mathrm{O}), 1717(\mathrm{C}=\mathrm{O}), 1606$ $(\mathrm{C}=\mathrm{N}), 777(\mathrm{C}-\mathrm{Br}) ;{ }^{1} \mathrm{H}$ NMR (400MHz, DMSO- $\left.d_{6}\right): \delta 1.29$ (t, 3H, $\left.J=7.6\right), 2.28(\mathrm{~s}, 3 \mathrm{H}), 2.78$ (s, $3 \mathrm{H}), 4.27-4.32(\mathrm{~m}, 2 \mathrm{H}), 7.47$ (d, 2H, $J=8.4 \mathrm{~Hz}), 7.52-7.63(\mathrm{~m}, 1 \mathrm{H}), 7.75(\mathrm{~s}, 1 \mathrm{H}), 7.88$ (d, 1H, $J$ $=7.6 \mathrm{~Hz}), 8.20(\mathrm{~d}, 1 \mathrm{H}, J=7.6 \mathrm{~Hz}), 8.29(\mathrm{t}, 1 \mathrm{H}, J=6.8 \mathrm{~Hz}), 8.47(\mathrm{~s}, 1 \mathrm{H}), 8.51(\mathrm{~d}, 1 \mathrm{H}, J=6.0$ $\mathrm{Hz}), 8.65(\mathrm{~s}, 1 \mathrm{H}), 8.72(\mathrm{~s}, 1 \mathrm{H})$, 11.51. MS (ESI) m/z: $678[\mathrm{M}+1]^{+}$; Anal. Calcd. for $\mathrm{C}_{30} \mathrm{H}_{21} \mathrm{BrN}_{4} \mathrm{O}_{6} \mathrm{~S}_{2}: \mathrm{C}, 53.18 ; \mathrm{H}, 3.12 ; \mathrm{N}, 8.27$. Found: $\mathrm{C}, 53.27 ; \mathrm{H}, 3.01 ; \mathrm{N}, 8.41$.

Ethyl-2-(3-(1-(2-(4-(6,8-dibromo-2-oxo-2H-chromen-3-yl)thiazol-2-yl)hydrazono)ethyl)-2oxo-2H-chromen-6-yl)-4-methylthiazole-5-carboxylate (6k)

Yellow solid; mp. 232-234 ${ }^{\circ} \mathrm{C}$; IR ( $\left.\mathrm{KBr}, \mathrm{cm}^{-1}\right) v_{\max }: 3417(\mathrm{NH}), 1736(\mathrm{C}=\mathrm{O}), 1715(\mathrm{C}=\mathrm{O}), 1619$ $(\mathrm{C}=\mathrm{N}), 1567(\mathrm{C}=\mathrm{C}), 778(\mathrm{C}-\mathrm{Br}) ;{ }^{1} \mathrm{H}$ NMR $\left(400 \mathrm{MHz}, \mathrm{DMSO}-d_{6}\right): \delta 1.33(\mathrm{t}, 3 \mathrm{H}, J=7.6 \mathrm{~Hz}), 2.30$ (s, 3H), 2.75 (s, 3H), 4.31-4.35 (m, 2H), $7.20(\mathrm{~d}, 2 \mathrm{H}, J=8.4 \mathrm{~Hz}), 7.28(\mathrm{~s}, 1 \mathrm{H}), 7.56(\mathrm{~d}, 1 \mathrm{H}, J=$ $8.4 \mathrm{~Hz}), 7.75$ (s, 1H), 8.16 (s, 1H), 8.26 (s, 1H), 8.54 (s, 1H), 11.39 (s, 1H); MS (ESI) m/z: 757 $[\mathrm{M}+1]^{+}$; Anal. Calcd. for $\mathrm{C}_{30} \mathrm{H}_{20} \mathrm{Br}_{2} \mathrm{~N}_{4} \mathrm{O}_{6} \mathrm{~S}_{2}: \mathrm{C}, 47.63 ; \mathrm{H}, 2.66 ; \mathrm{N}, 7.41$. Found: $\mathrm{C}, 47.47 ; \mathrm{H}, 2.76$; N, 7.32.

Ethyl-4-methyl-2-(2-oxo-3-(1-(2-(4-(3-0xo-3H-benzo[f]chromen-2-yl)thiazol-2yl)hydrazono)ethyl)-2H-chromen-6-yl)thiazole-5-carboxylate (6l)

Bright yellow solid; mp. 242-244 ${ }^{\circ} \mathrm{C}$; IR $\left(\mathrm{KBr}, \mathrm{cm}^{-1}\right) v_{\max }: 3416(\mathrm{NH}), 1719(\mathrm{C}=\mathrm{O}), 1615(\mathrm{C}=\mathrm{N})$, $1561(\mathrm{C}=\mathrm{C}) ;{ }^{1} \mathrm{H}$ NMR $\left(400 \mathrm{MHz}, \mathrm{DMSO}-d_{6}\right): \delta 1.31(\mathrm{t}, 3 \mathrm{H}, J=7.6 \mathrm{~Hz}), 2.31(\mathrm{~s}, 3 \mathrm{H}), 2.72(\mathrm{~s}$, $3 \mathrm{H}), 4.27-4.33(\mathrm{~m}, 2 \mathrm{H}), 7.56(\mathrm{~d}, 2 \mathrm{H}, J=8.8 \mathrm{~Hz}), 7.64-7.68(\mathrm{~m}, 4 \mathrm{H}), 7.85(\mathrm{~d}, 2 \mathrm{H}, J=8.0 \mathrm{~Hz})$, $8.11(\mathrm{~d}, 1 \mathrm{H}, J=7.6 \mathrm{~Hz}), 8.23$ (t, 1H, $J=8.4 \mathrm{~Hz}), 8.34$ (s, 1H), 8.60 (s, 1H), 11.48 (s, 1H); MS (ESI) $m / z$ : $649[\mathrm{M}+1]^{+}$; Anal. Calcd. for $\mathrm{C}_{34} \mathrm{H}_{24} \mathrm{~N}_{4} \mathrm{O}_{6} \mathrm{~S}_{2}: \mathrm{C}, 62.95 ; \mathrm{H}, 3.73 ; \mathrm{N}, 8.64$. Found: $\mathrm{C}$, $63.05 ; \mathrm{H}, 3.59 ; \mathrm{N}, 8.74$. 
Spectra of synthesized compounds

${ }^{1}$ H NMR (400 MHz, DMSO- $d_{6}$ ) spectrum of compound 3

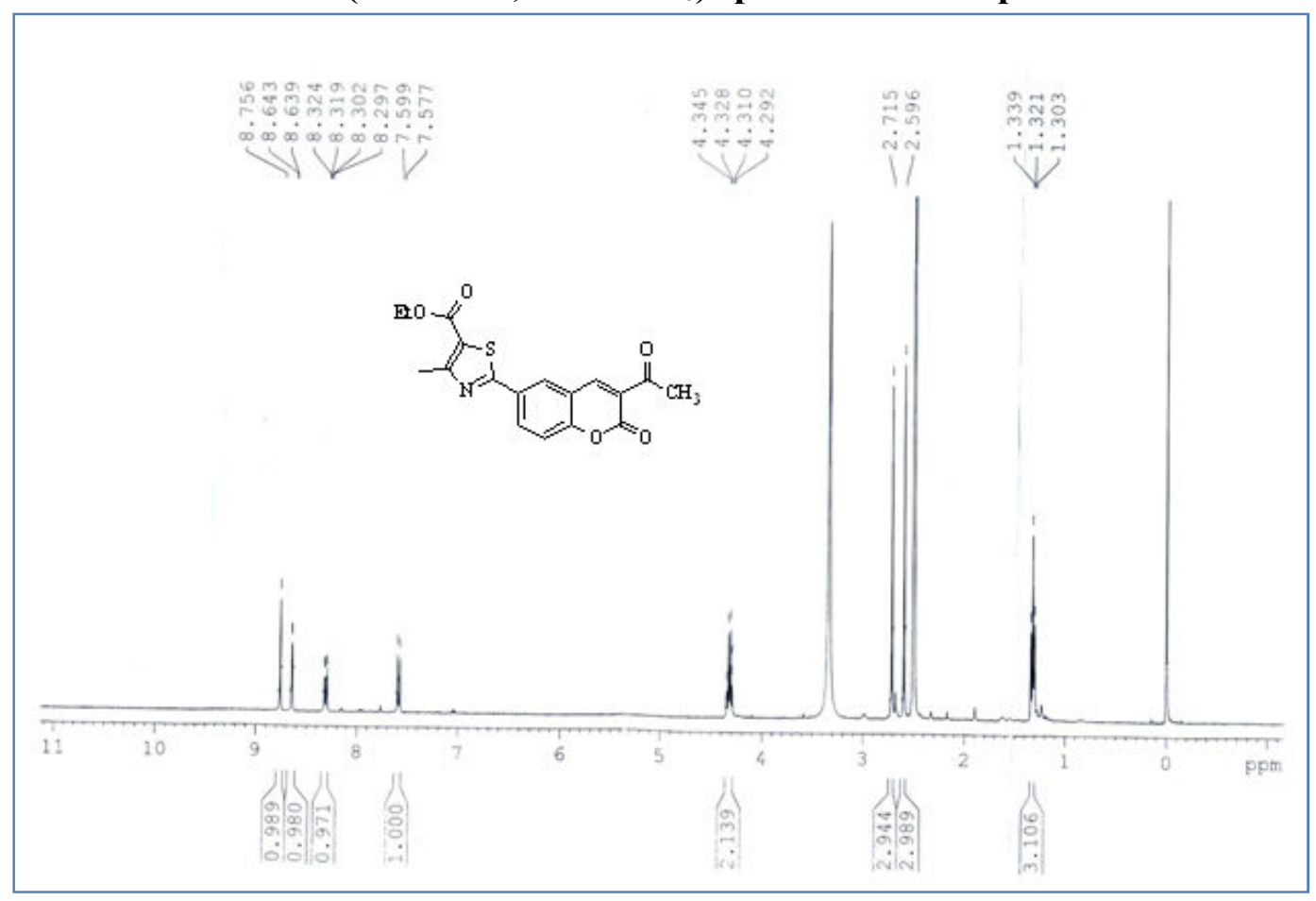

${ }^{13}$ C NMR (100 MHz, DMSO- $\left.d_{6}\right)$ spectrum of compound 3

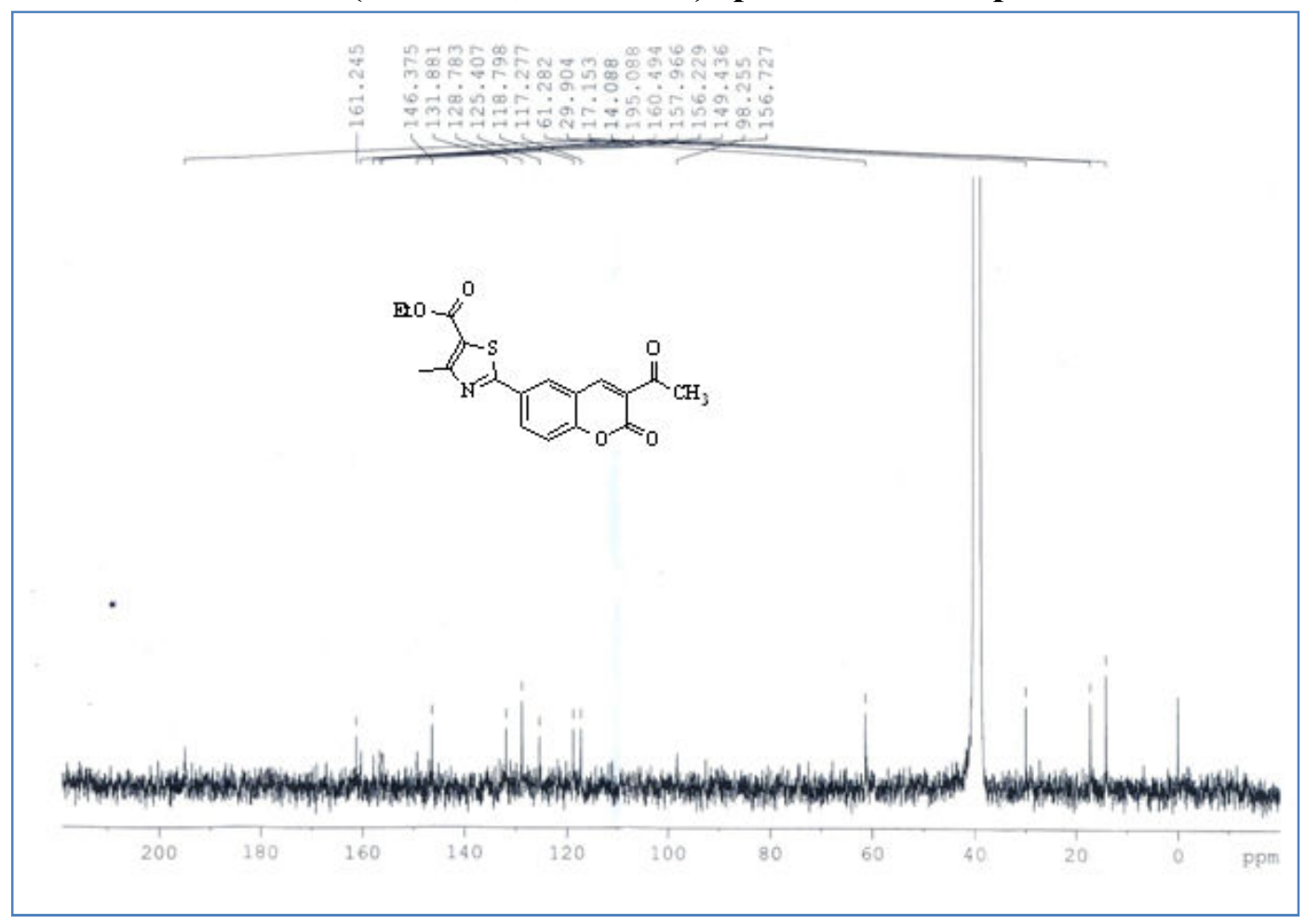




\section{IR (KBr) spectrum of compound 6a}

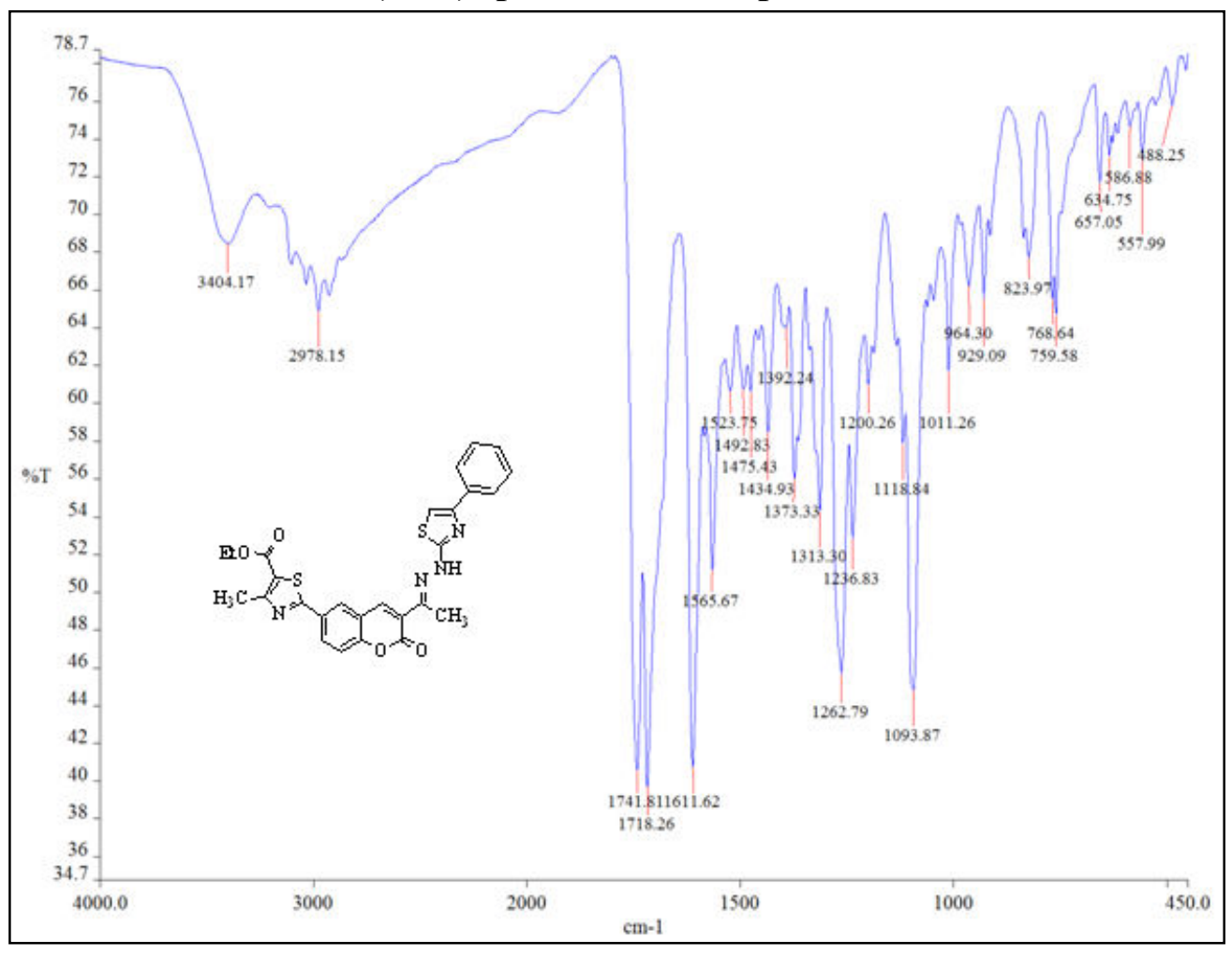

\section{${ }^{1} \mathrm{H}$ NMR (400 MHz, DMSO- $d_{6}$ ) spectrum of compound $6 \mathrm{a}$}

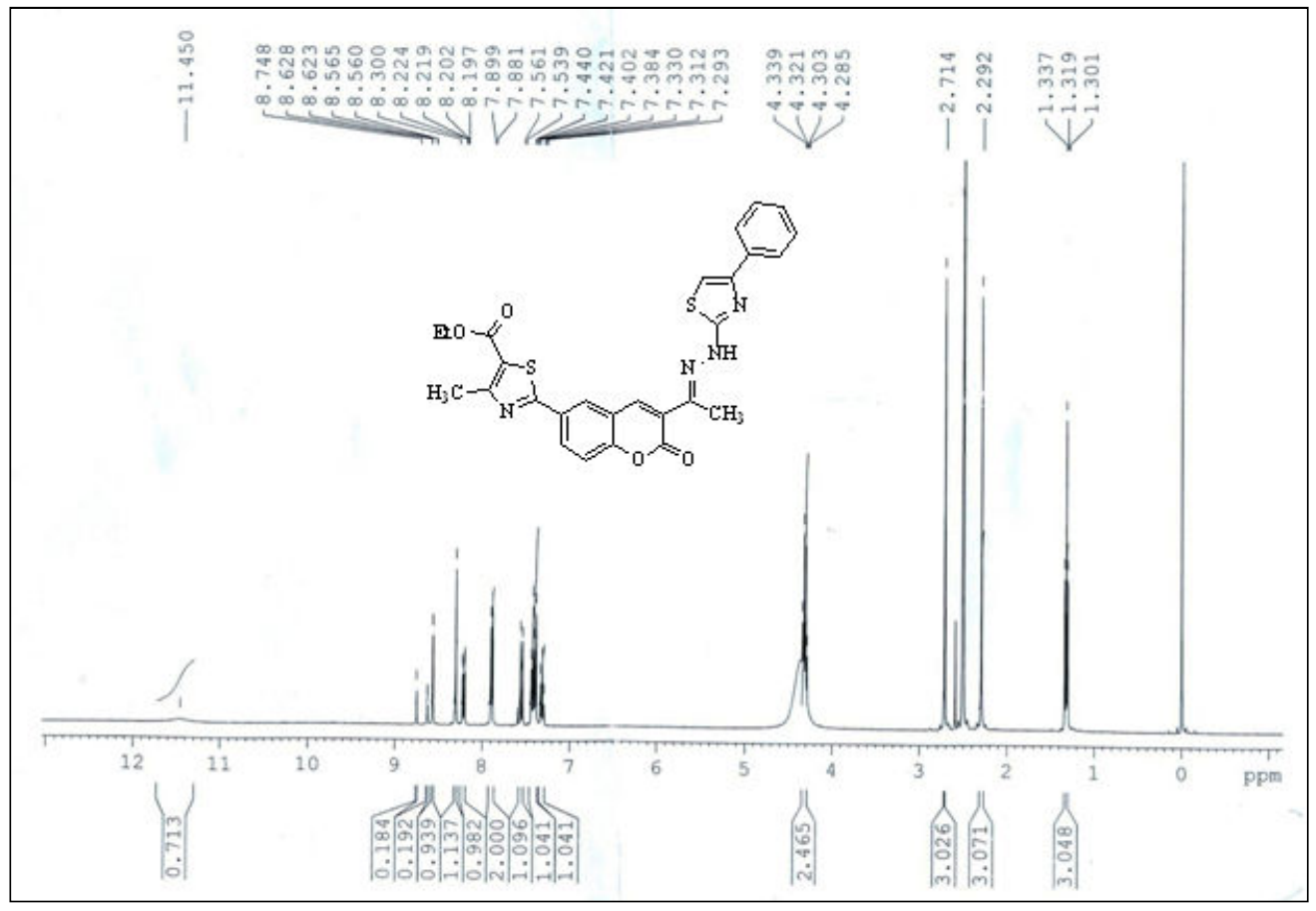


${ }^{13}$ C NMR (100 MHz, DMSO- $\left.d_{6}\right)$ spectrum of compound $6 a$

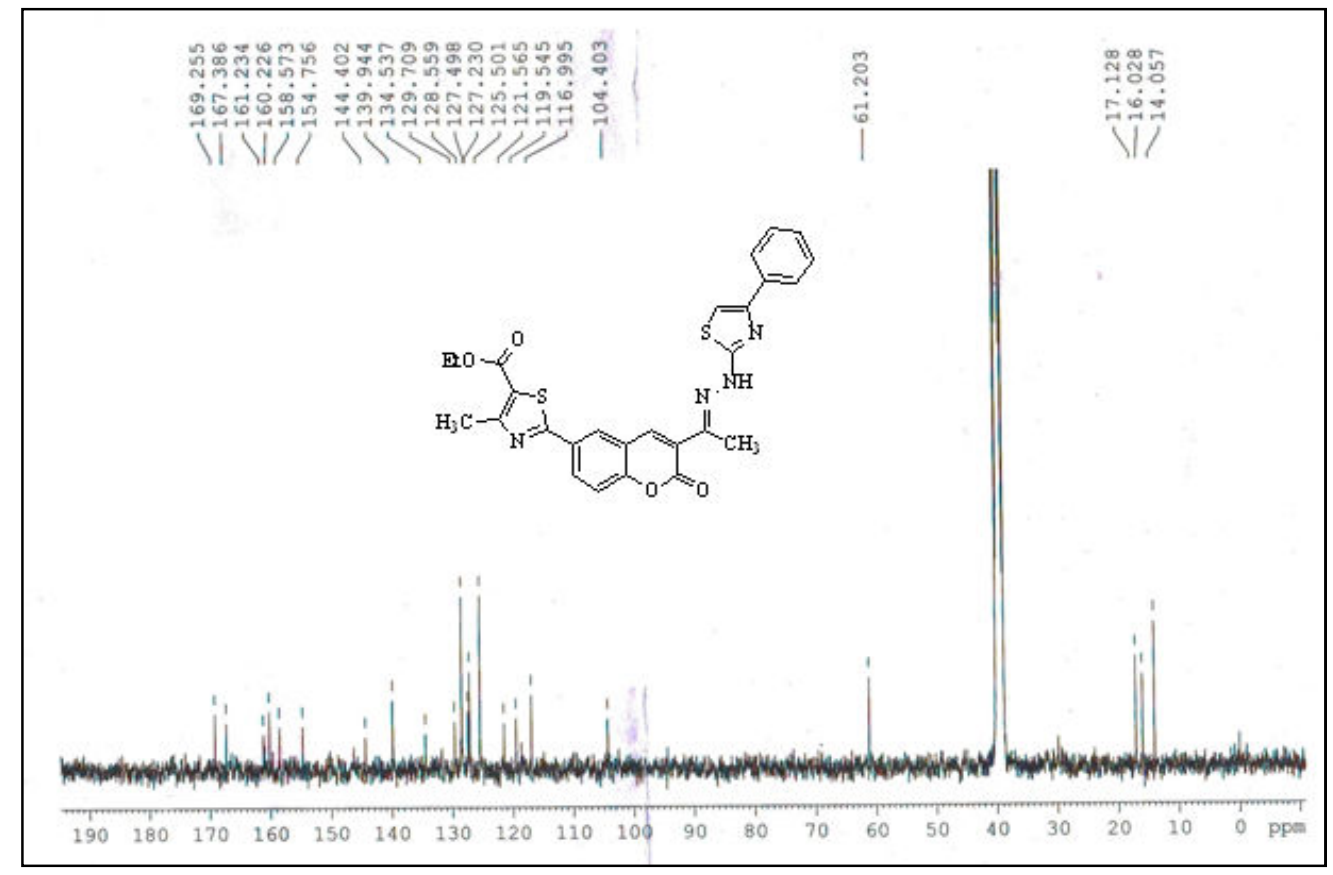

Mass spectrum of compound 6 a

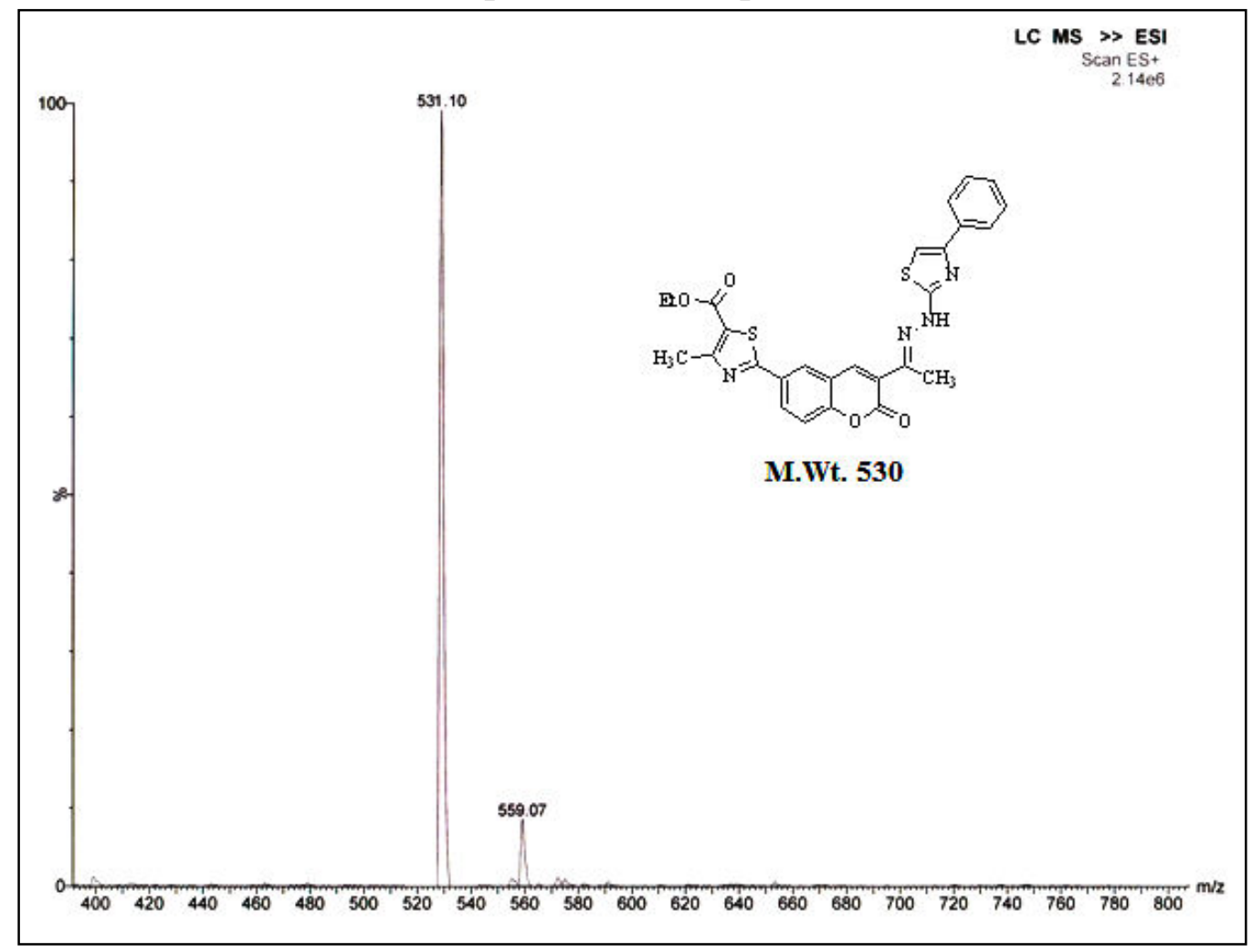




\section{IR (KBr) spectrum of compound $6 \mathrm{~b}$}

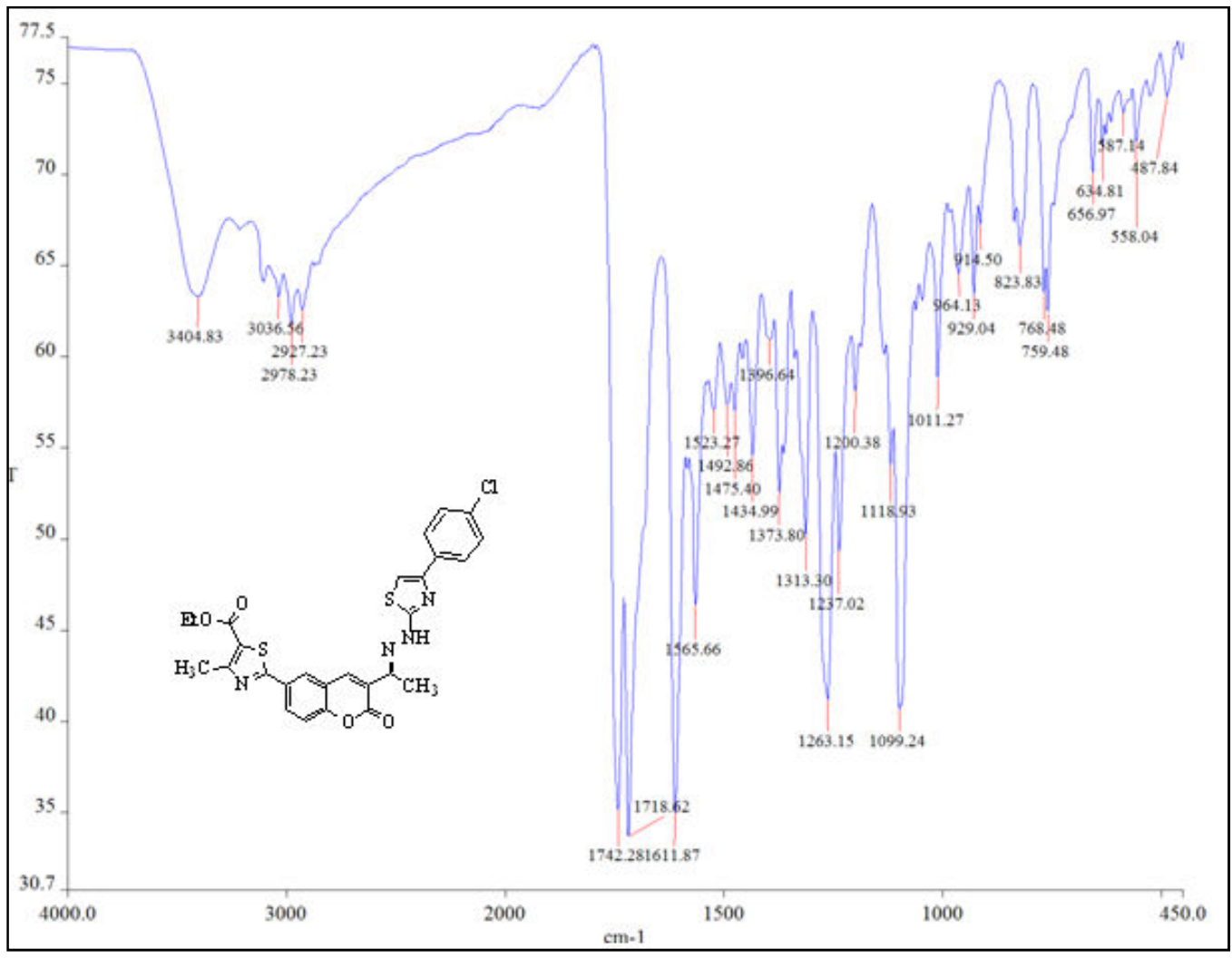

${ }^{1}$ H NMR (400 MHz, DMSO- $d_{6}$ ) spectrum of compound $6 \mathrm{~b}$

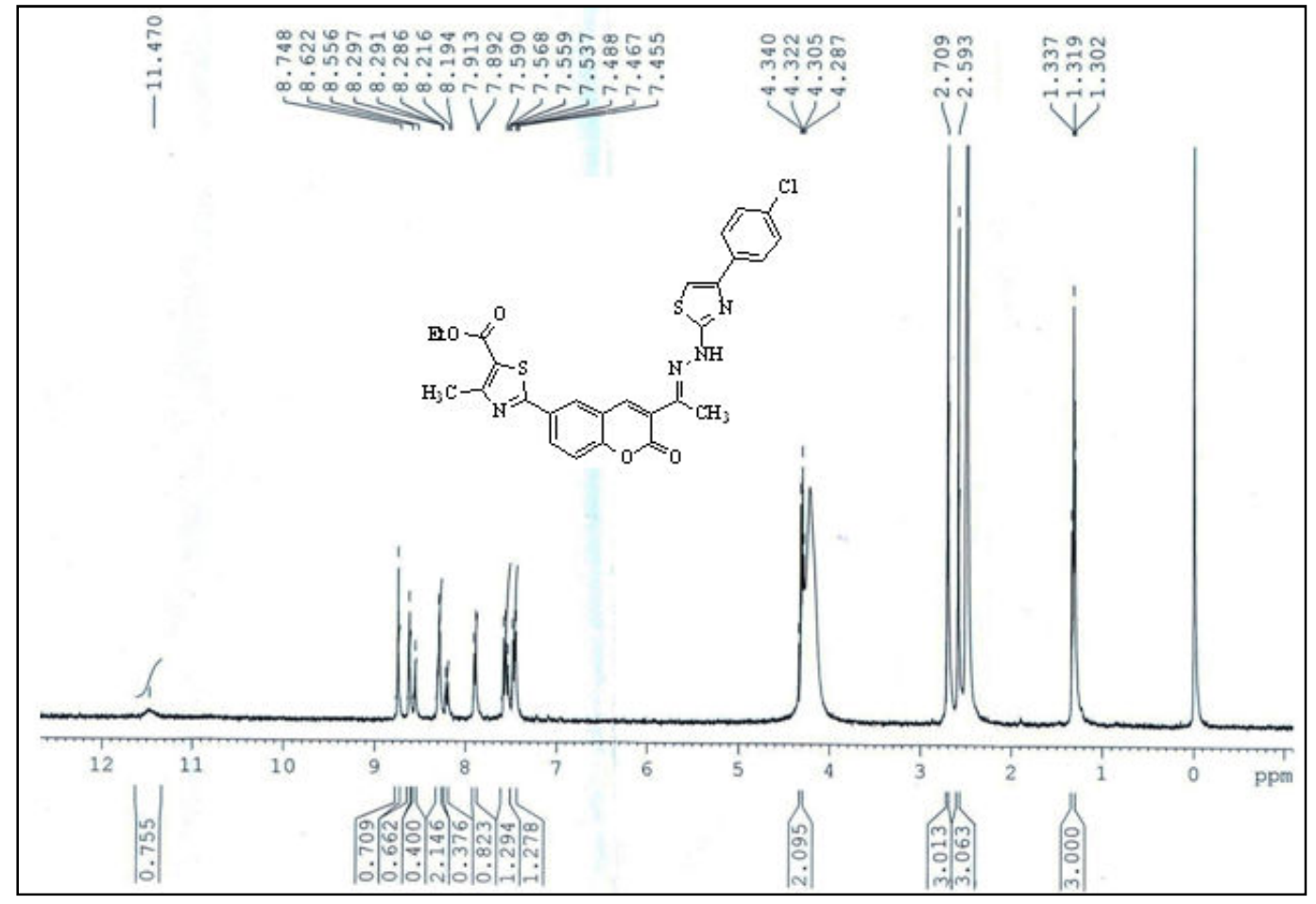


${ }^{13} \mathrm{C}$ NMR (100 MHz, DMSO- $\left.d_{6}\right)$ spectrum of compound $6 \mathrm{~b}$
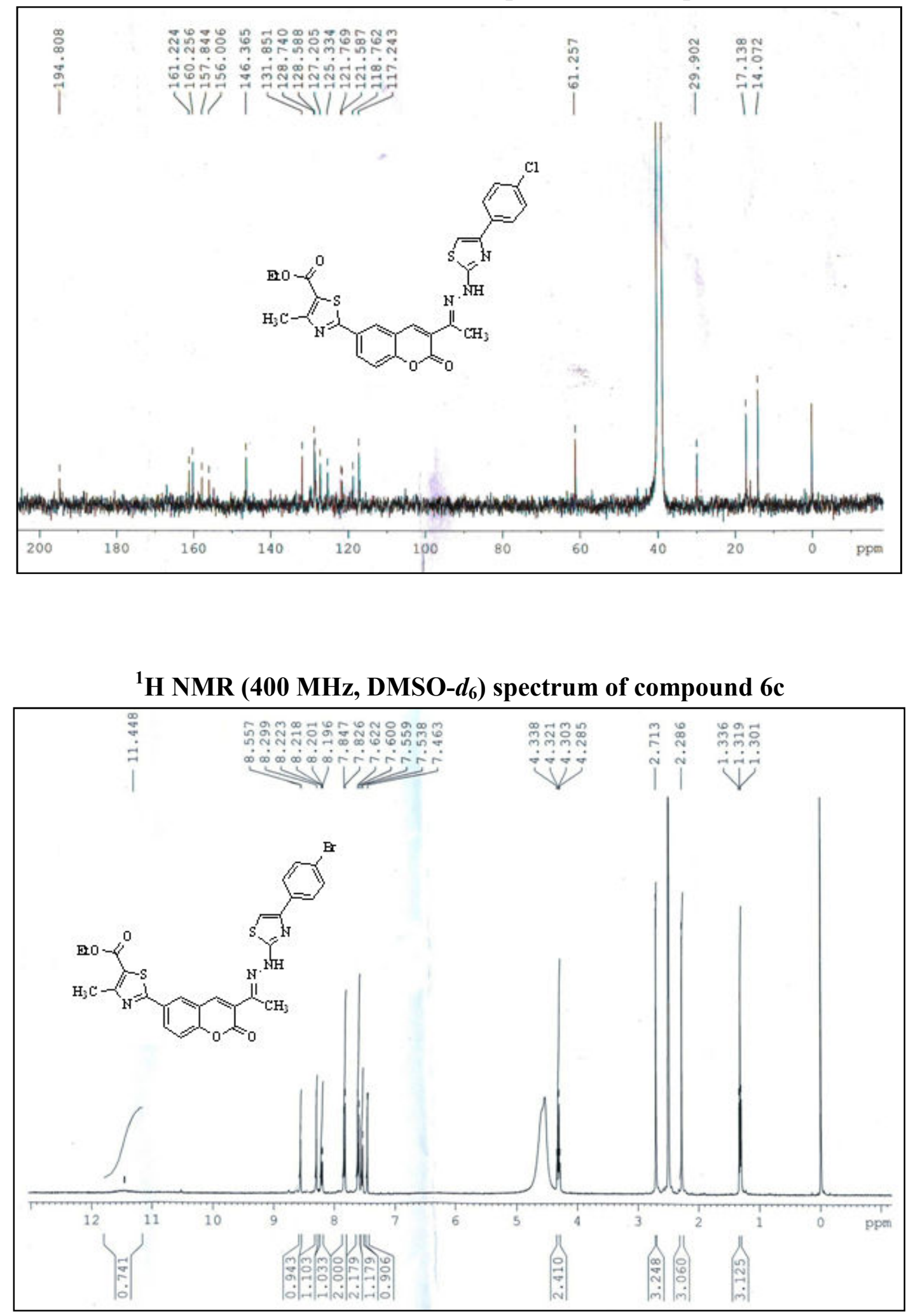


\section{${ }^{13} \mathrm{C}$ NMR (100 MHz, DMSO- $d_{6}$ ) spectrum of compound $6 \mathrm{c}$}

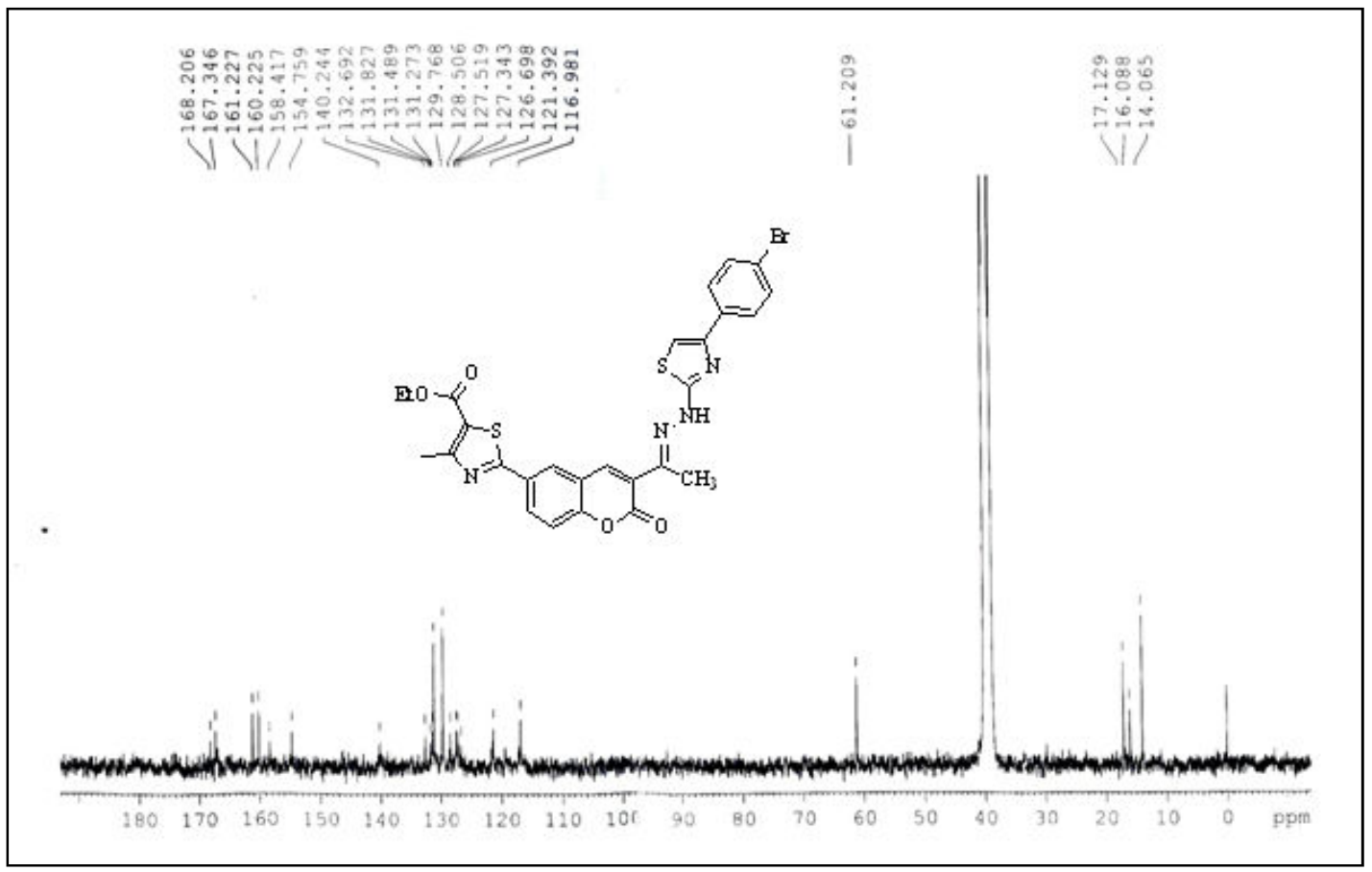

\section{IR (KBr) spectrum of compound $6 \mathrm{~d}$}

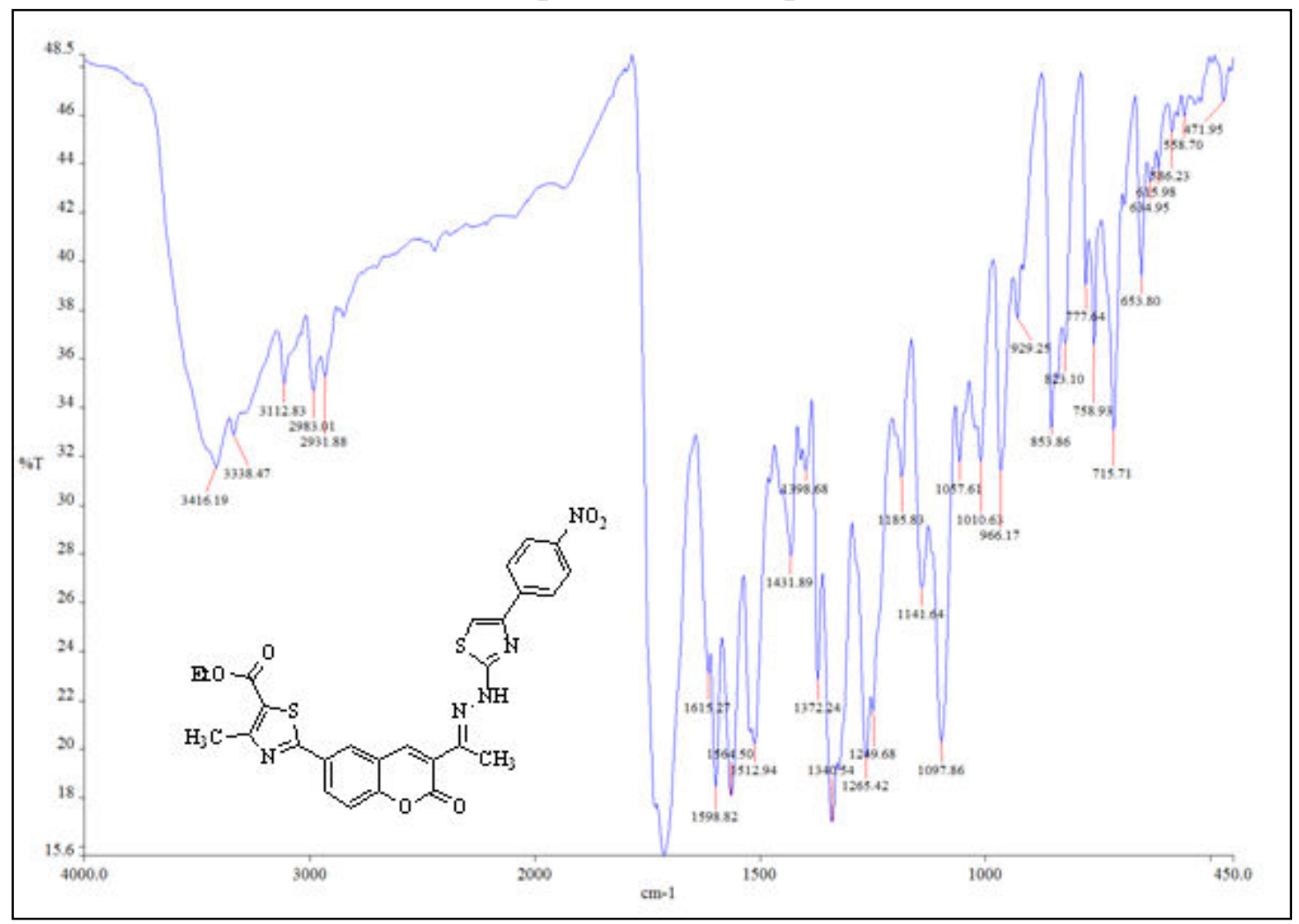


${ }^{1}$ H NMR (400 MHz, DMSO- $d_{6}$ ) spectrum of compound 6d

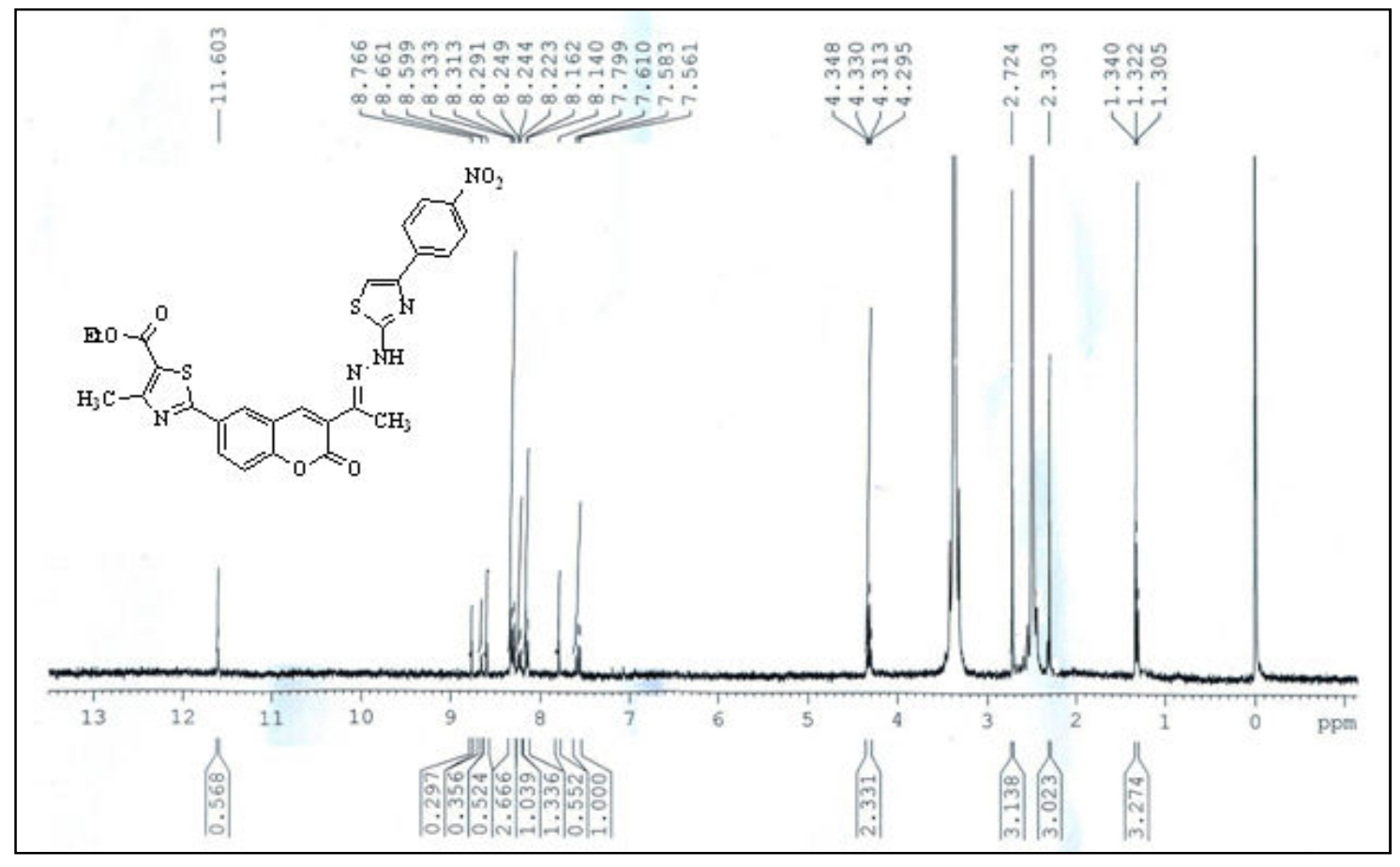

${ }^{13}$ C NMR (100 MHz, DMSO- $\left.d_{6}\right)$ spectrum of compound 6d

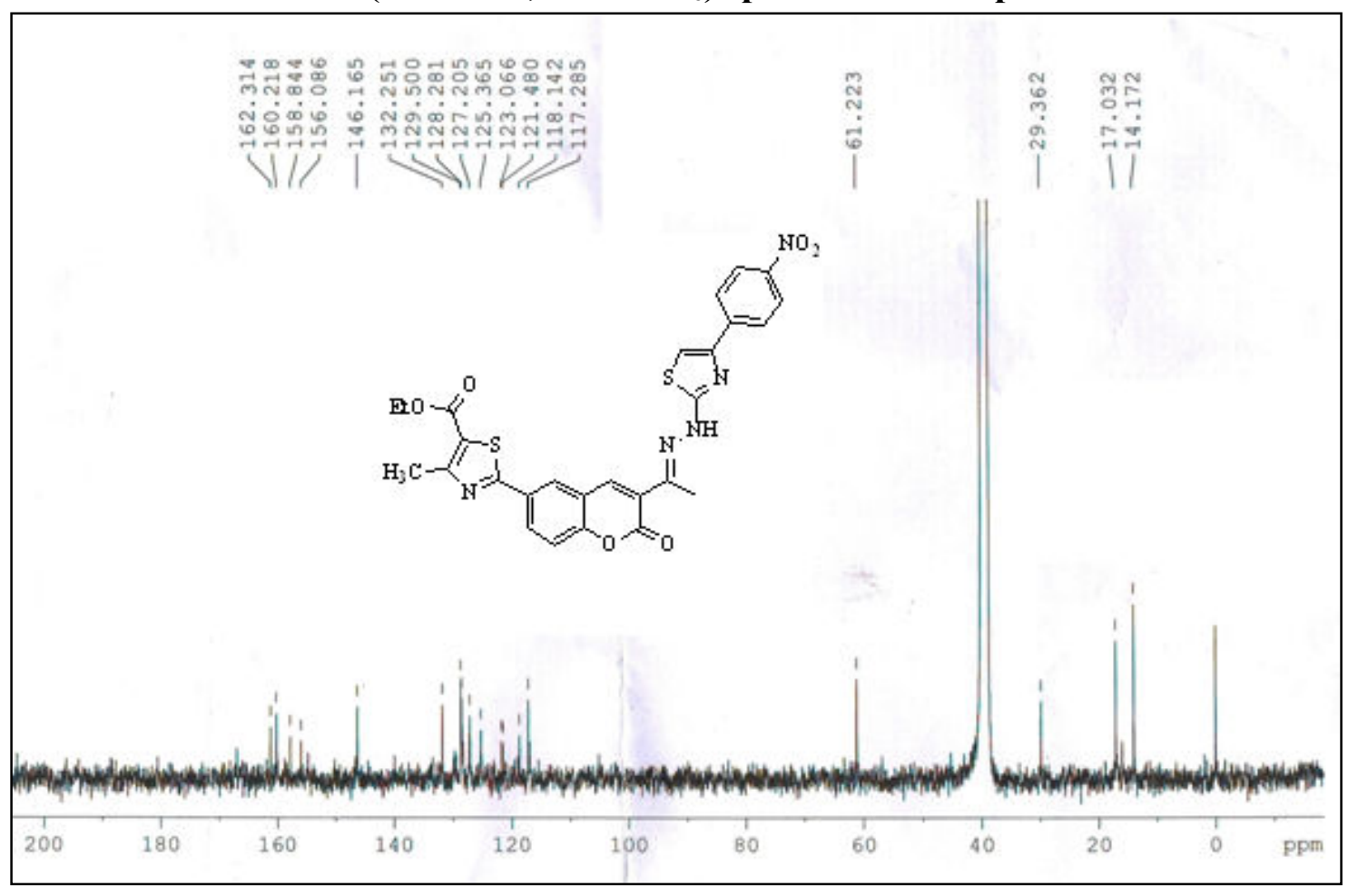




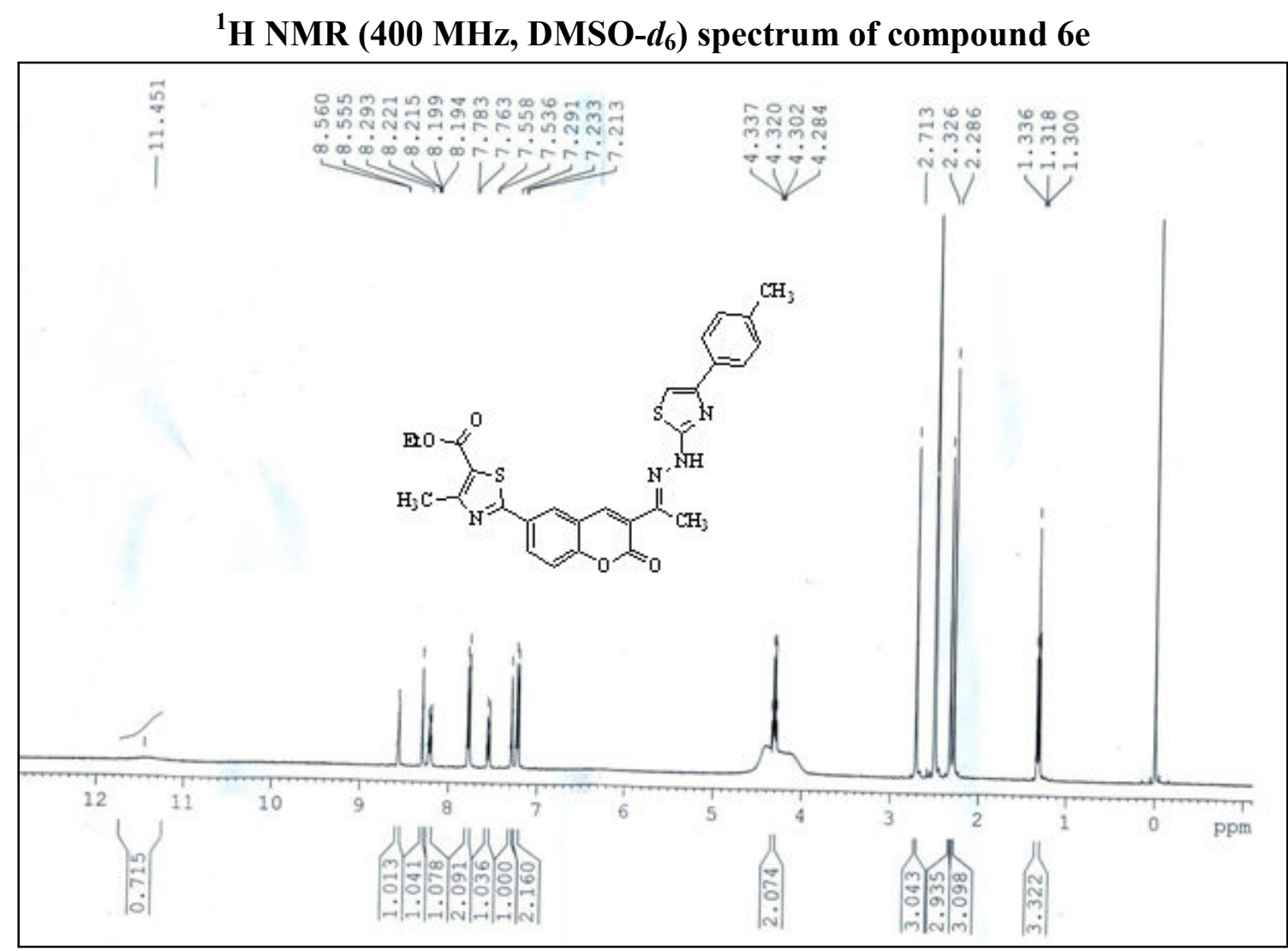

${ }^{13} \mathrm{C}$ NMR (100 MHz, DMSO-d ${ }_{6}$ ) spectrum of compound $6 \mathrm{e}$

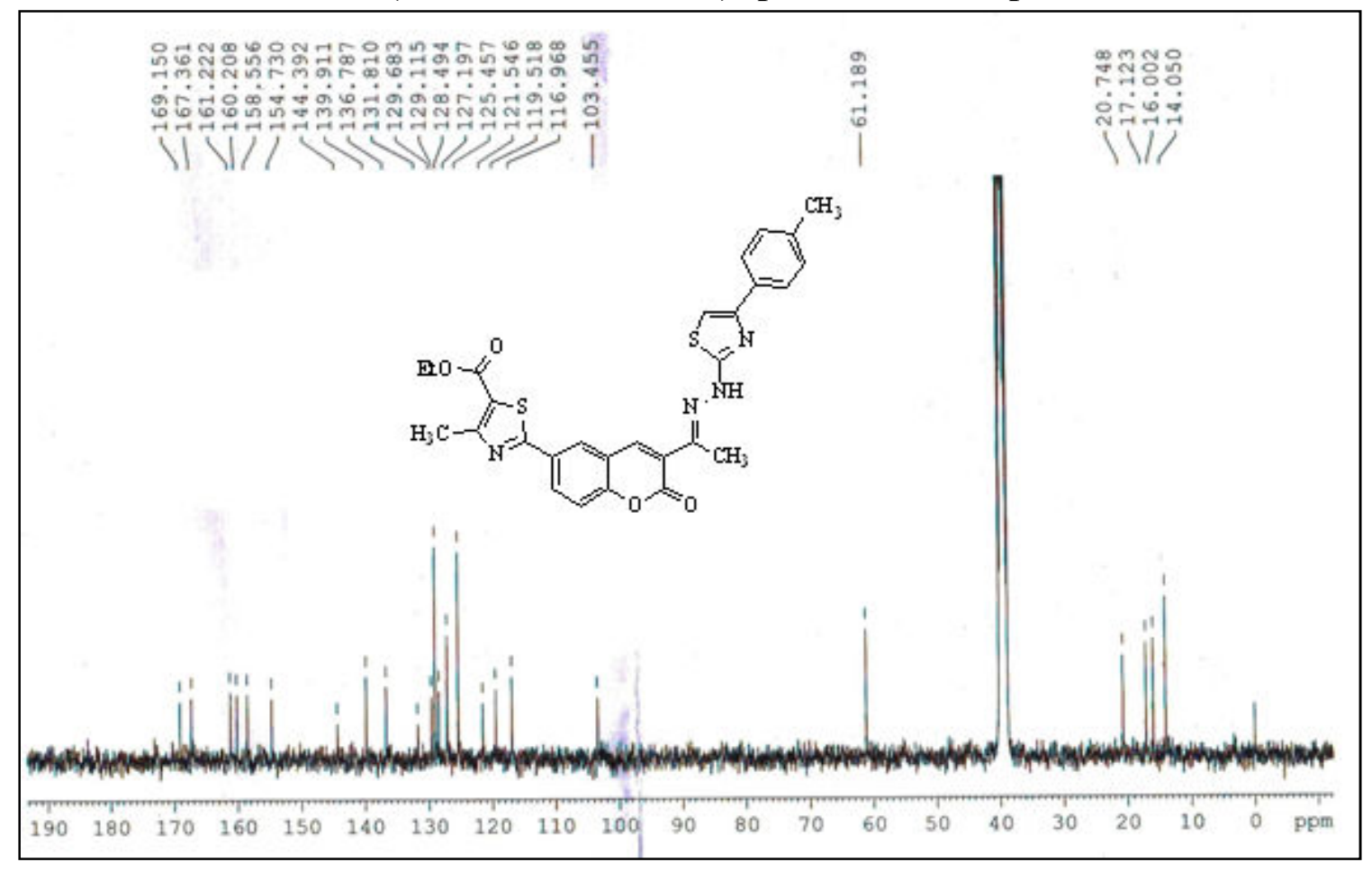




\section{Mass spectrum of compound $6 \mathrm{e}$}

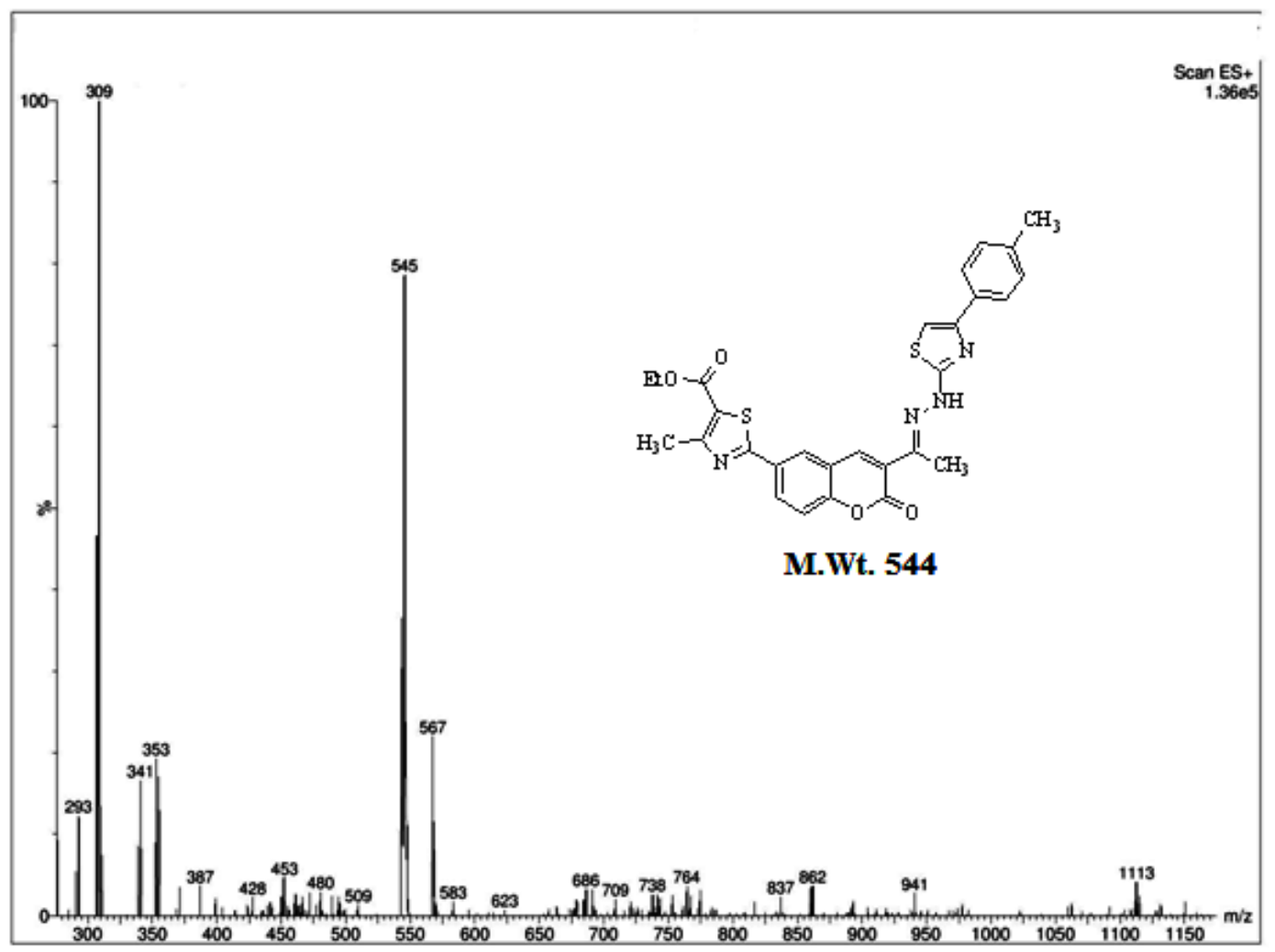

${ }^{13} \mathrm{C}$ NMR (100 MHz, DMSO- $\left.d_{6}\right)$ spectrum of compound $6 f$

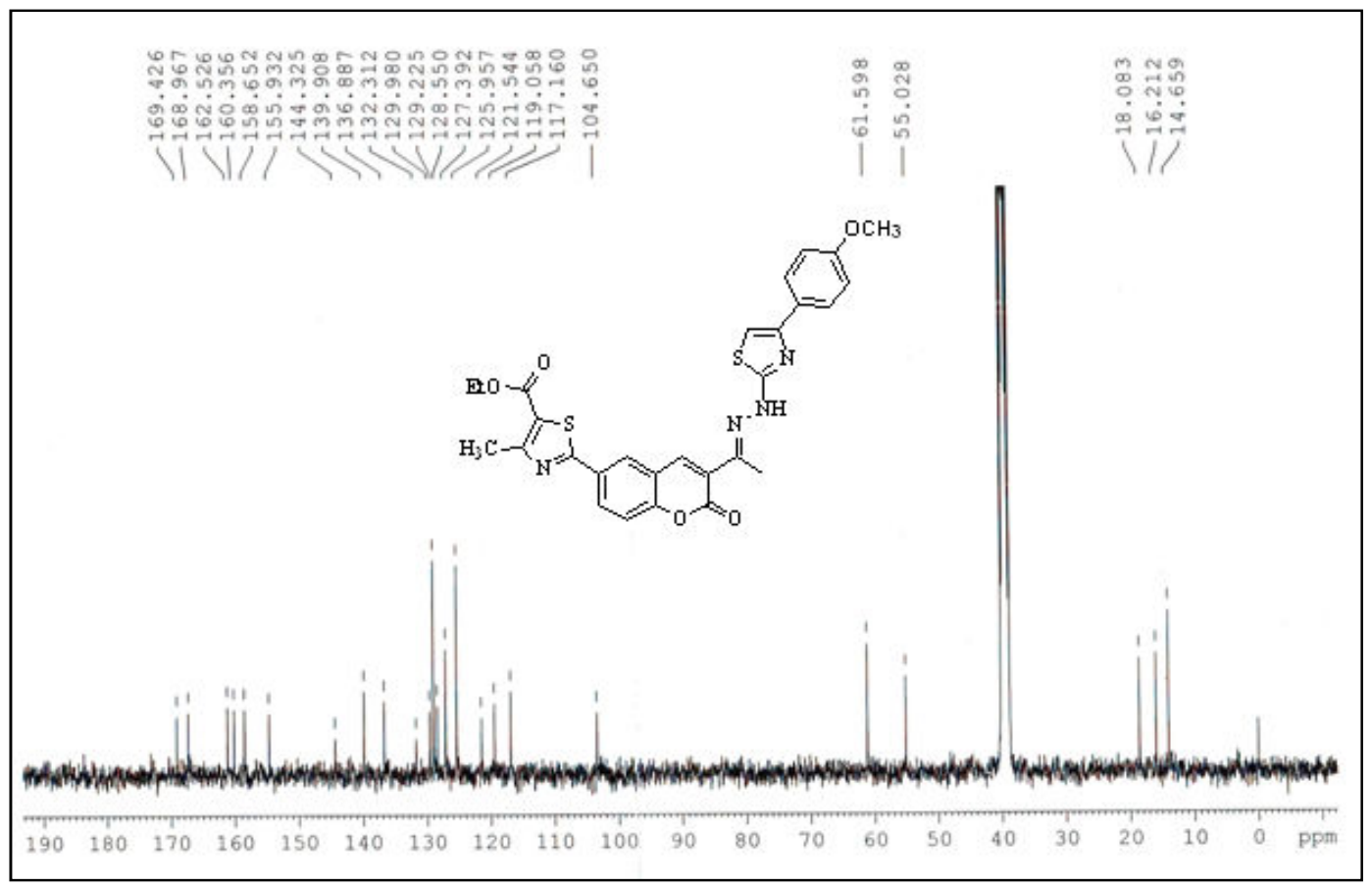




\section{IR (KBr) spectrum of compound $6 f$}

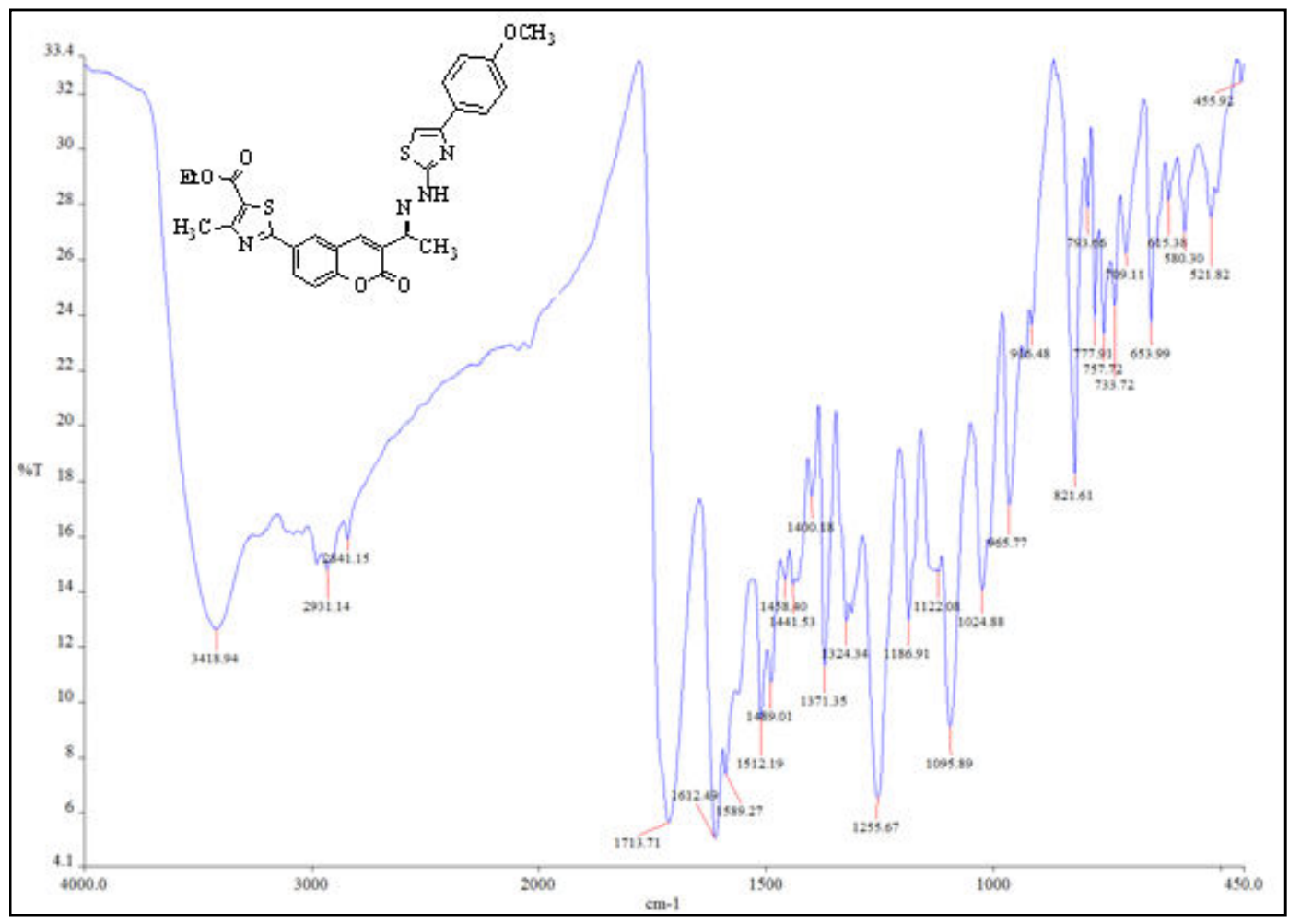

${ }^{1}$ H NMR (400 MHz, DMSO- $\left.d_{6}\right)$ spectrum of compound $6 \mathrm{~g}$

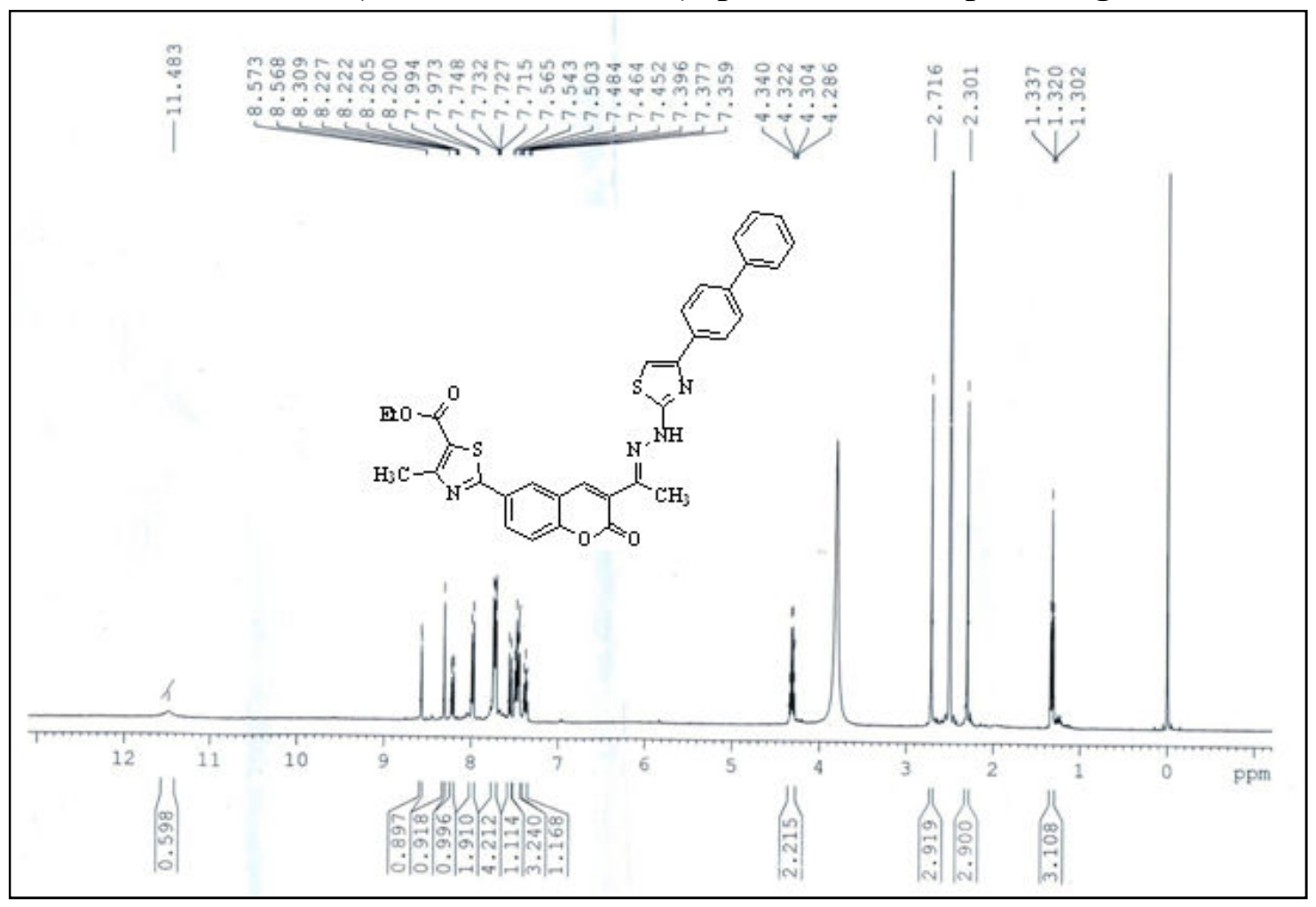




\section{IR (KBr) spectrum of compound $6 \mathrm{~h}$}

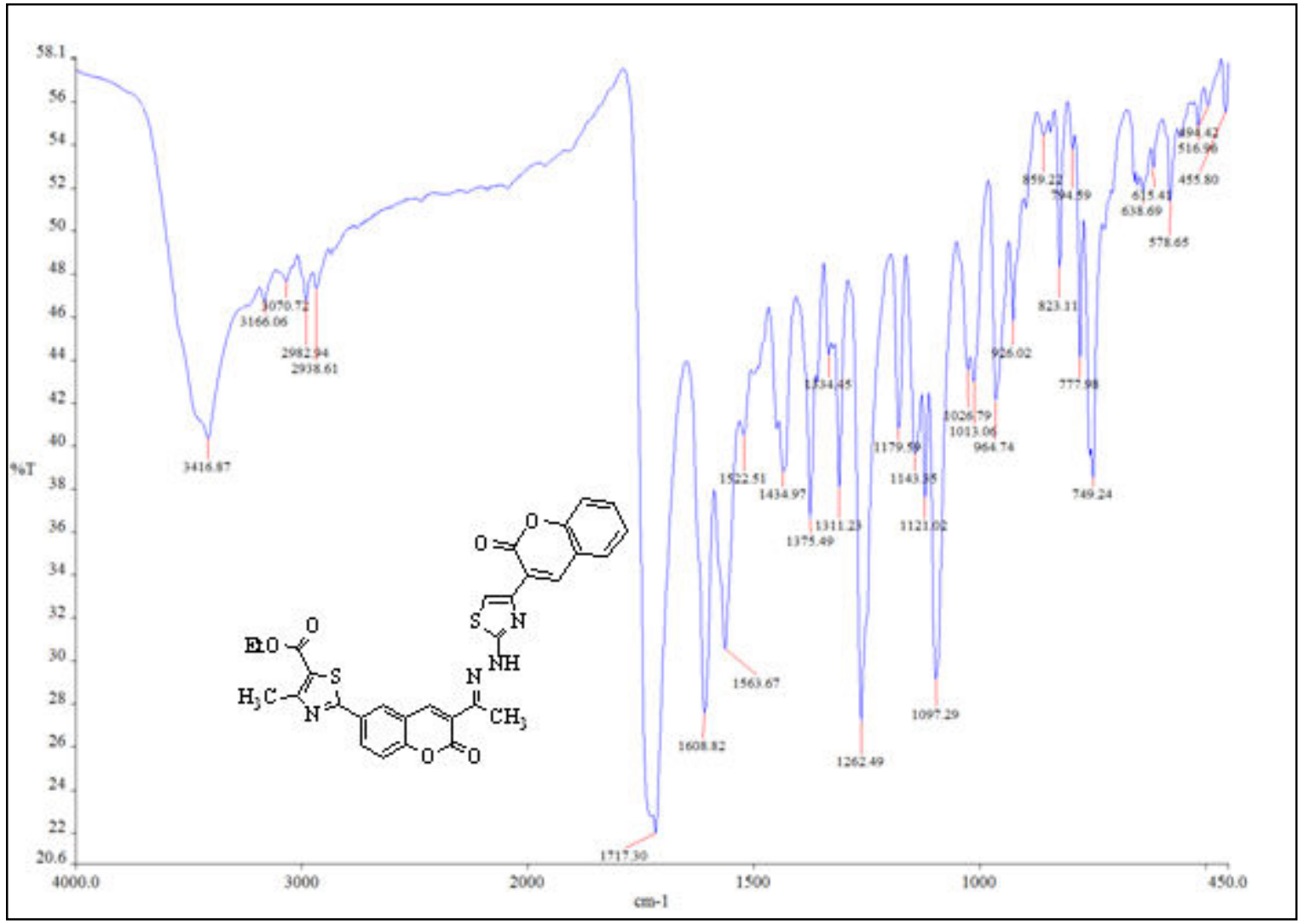

${ }^{1}$ H NMR (400 MHz, DMSO- $d_{6}$ ) spectrum of compound $6 \mathrm{~h}$

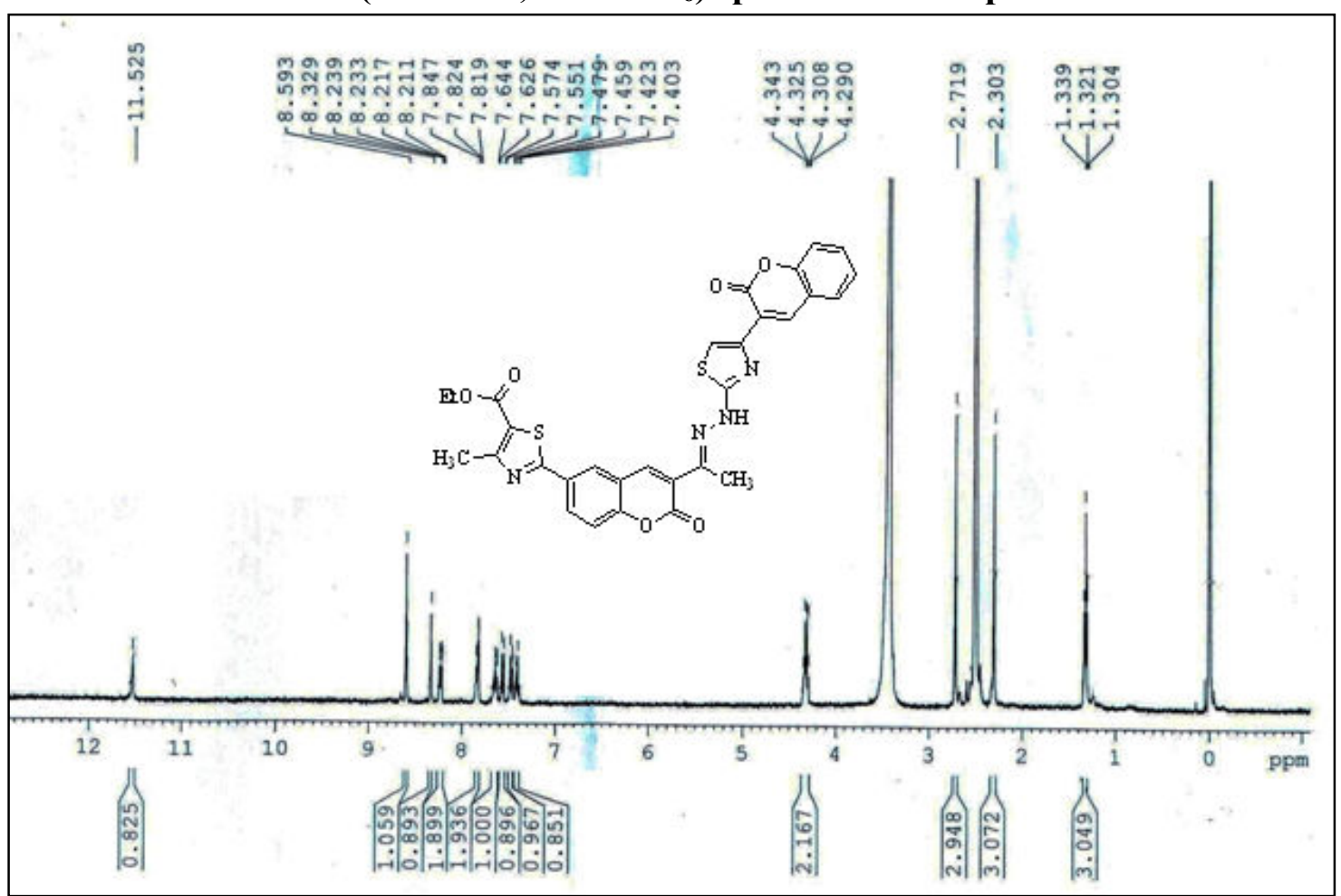




\section{${ }^{13} \mathrm{C}$ NMR (100 MHz, DMSO- $\left.d_{6}\right)$ spectrum of compound $6 \mathrm{~h}$}

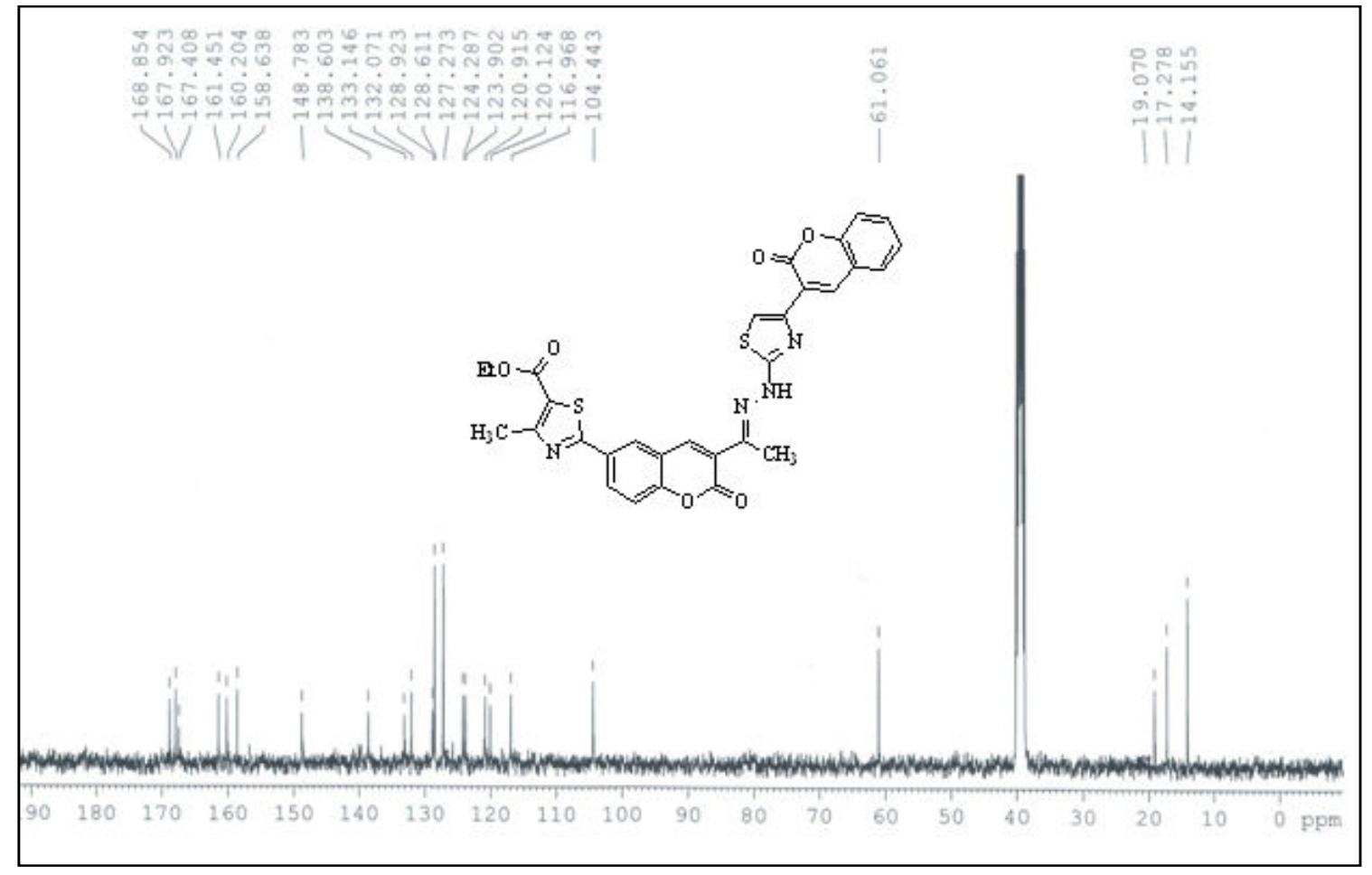

Mass spectrum of compound $6 \mathrm{~h}$

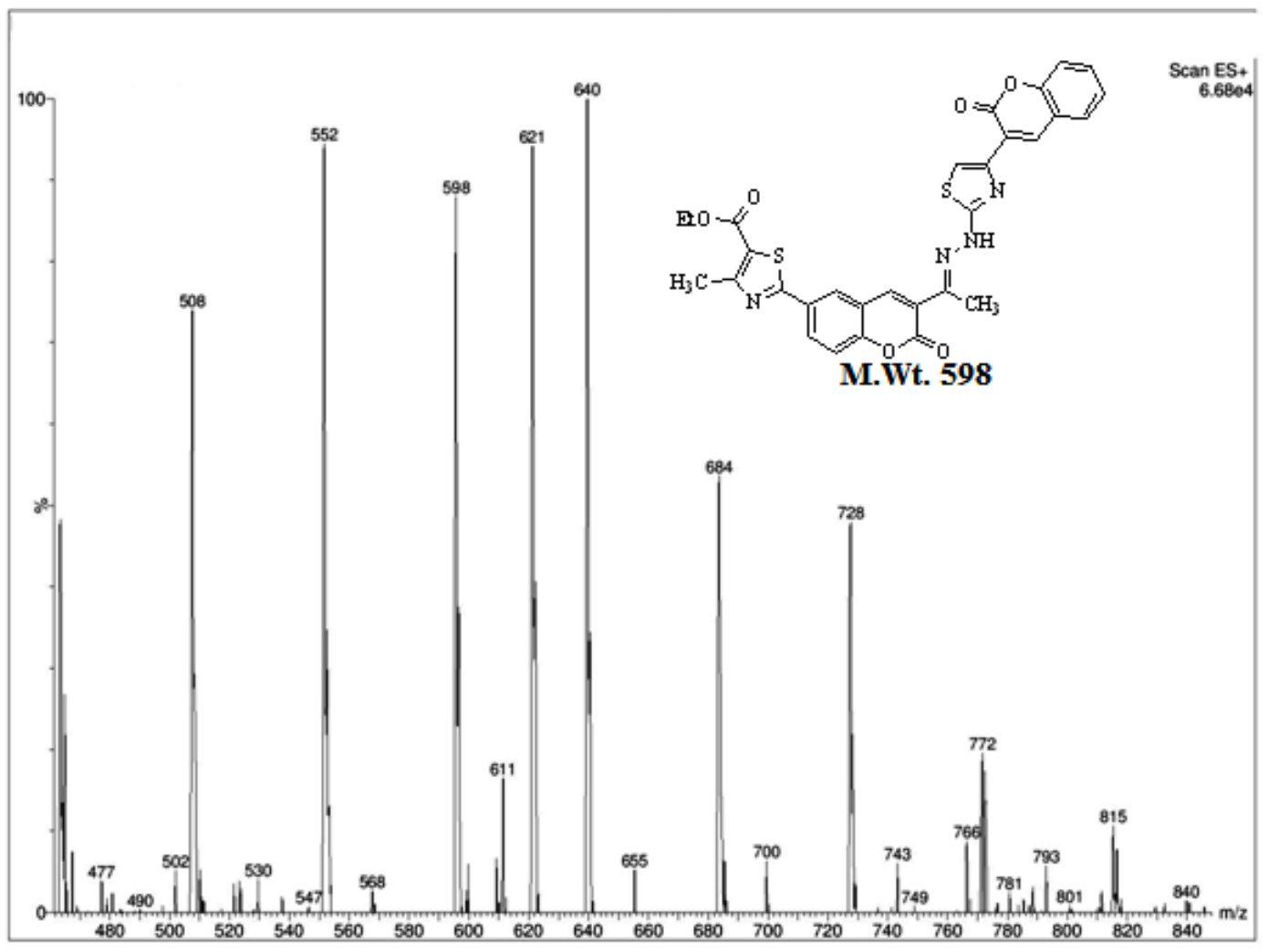




\section{IR (KBr) spectrum of compound $6 i$}

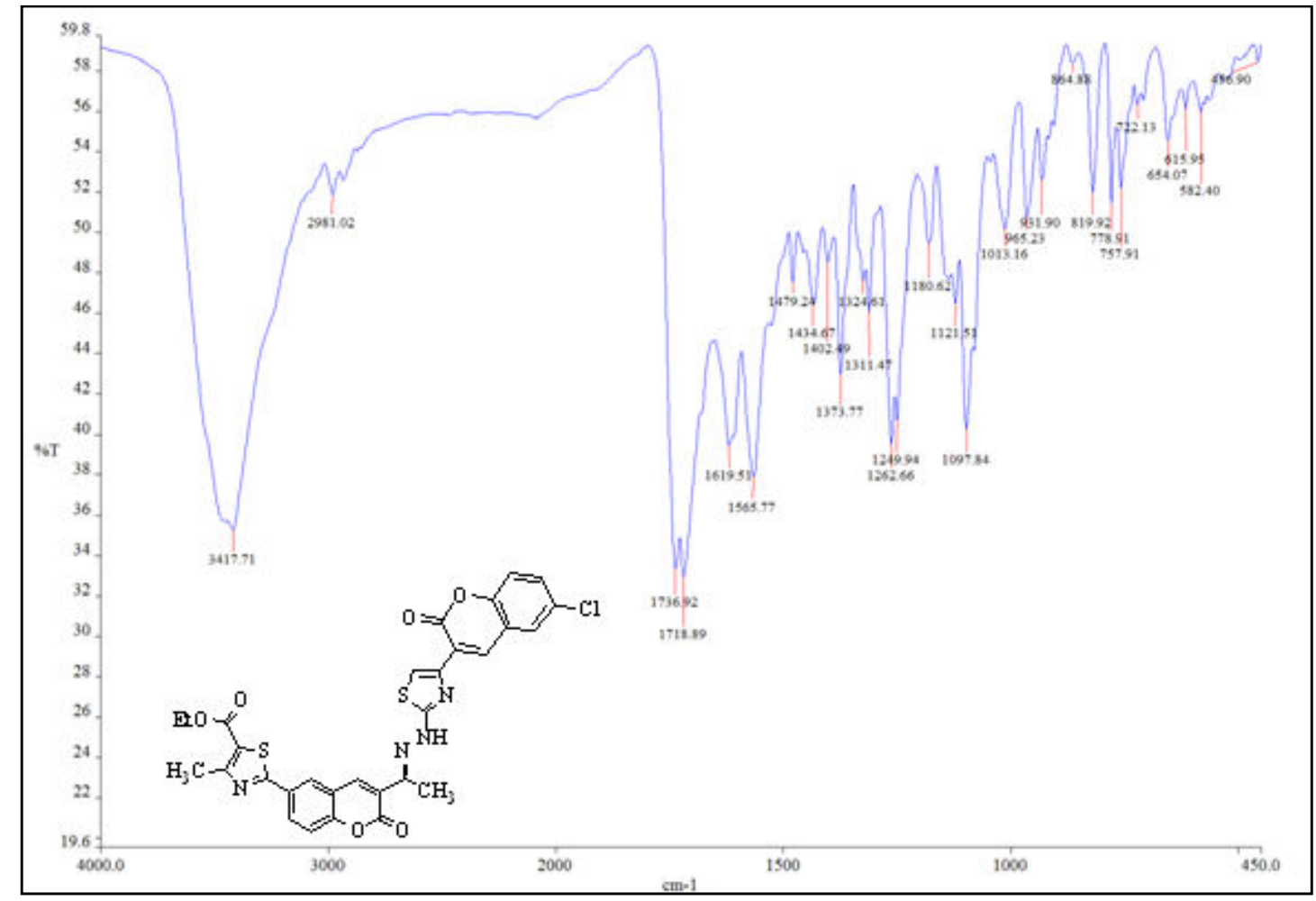

\section{${ }^{1}$ H NMR (400 MHz, DMSO- $d_{6}$ ) spectrum of compound $6 \mathrm{i}$}

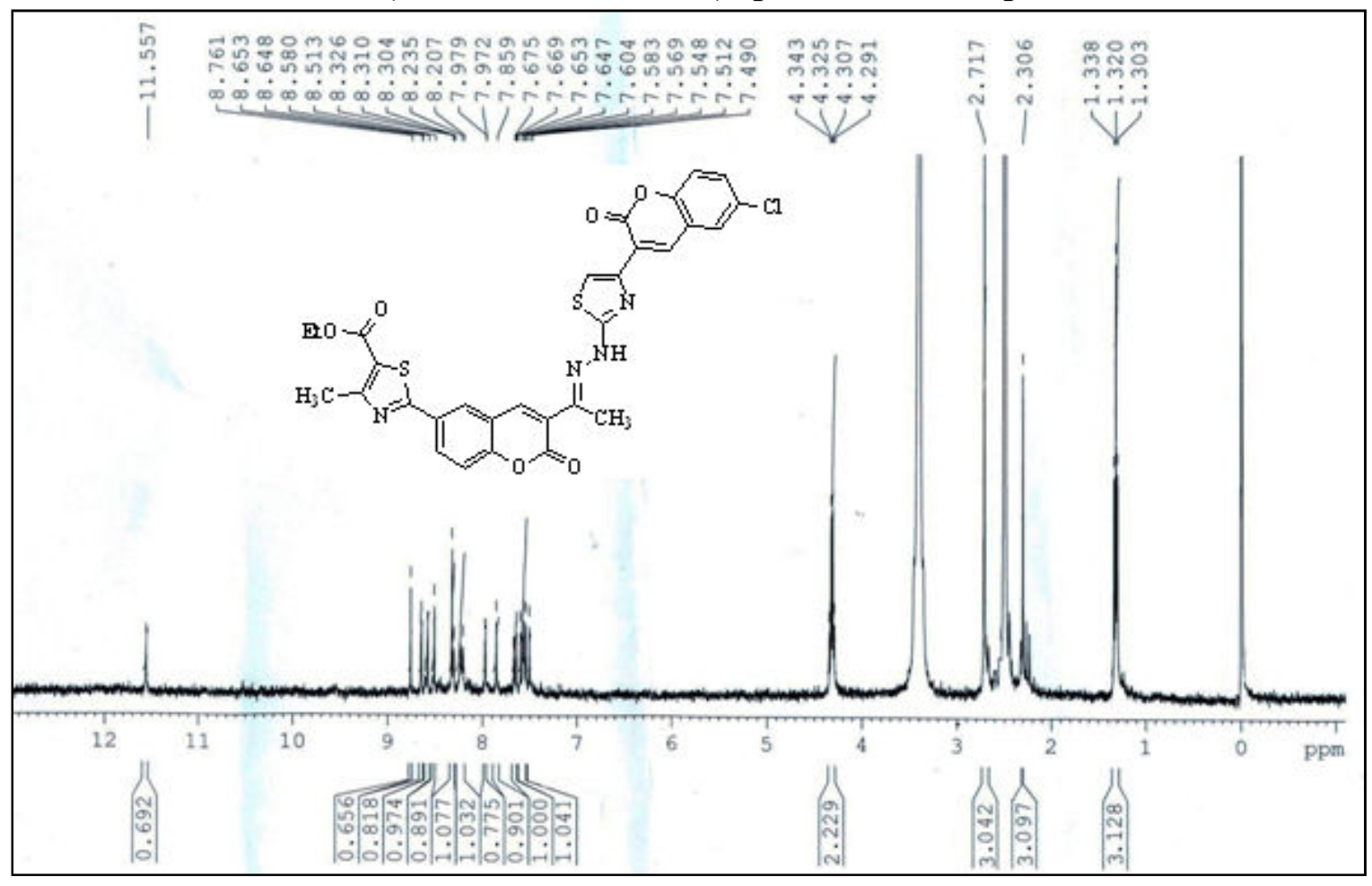




\section{IR (KBr) spectrum of compound $6 \mathbf{j}$}

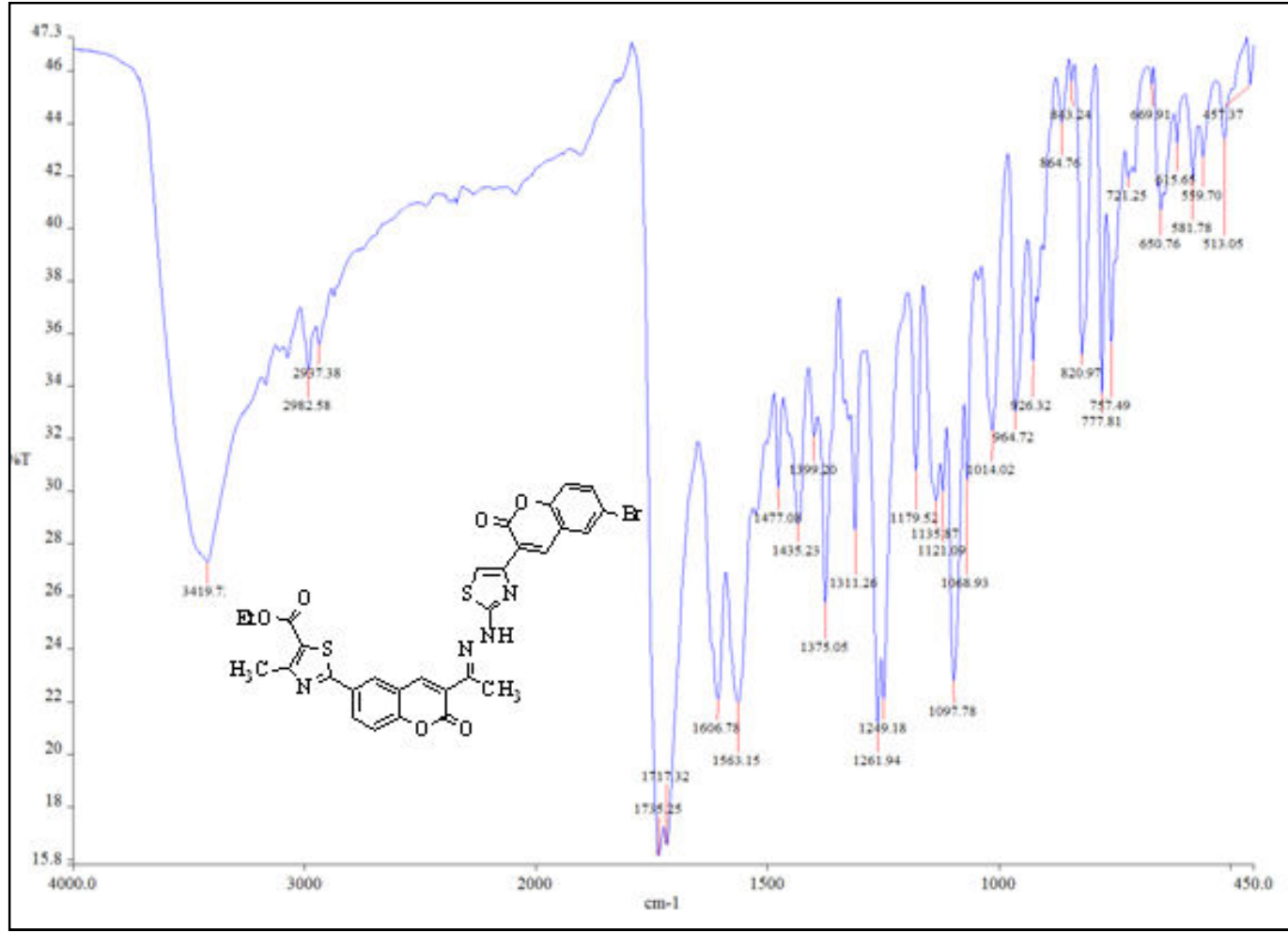

IR (KBr) spectrum of compound 6k

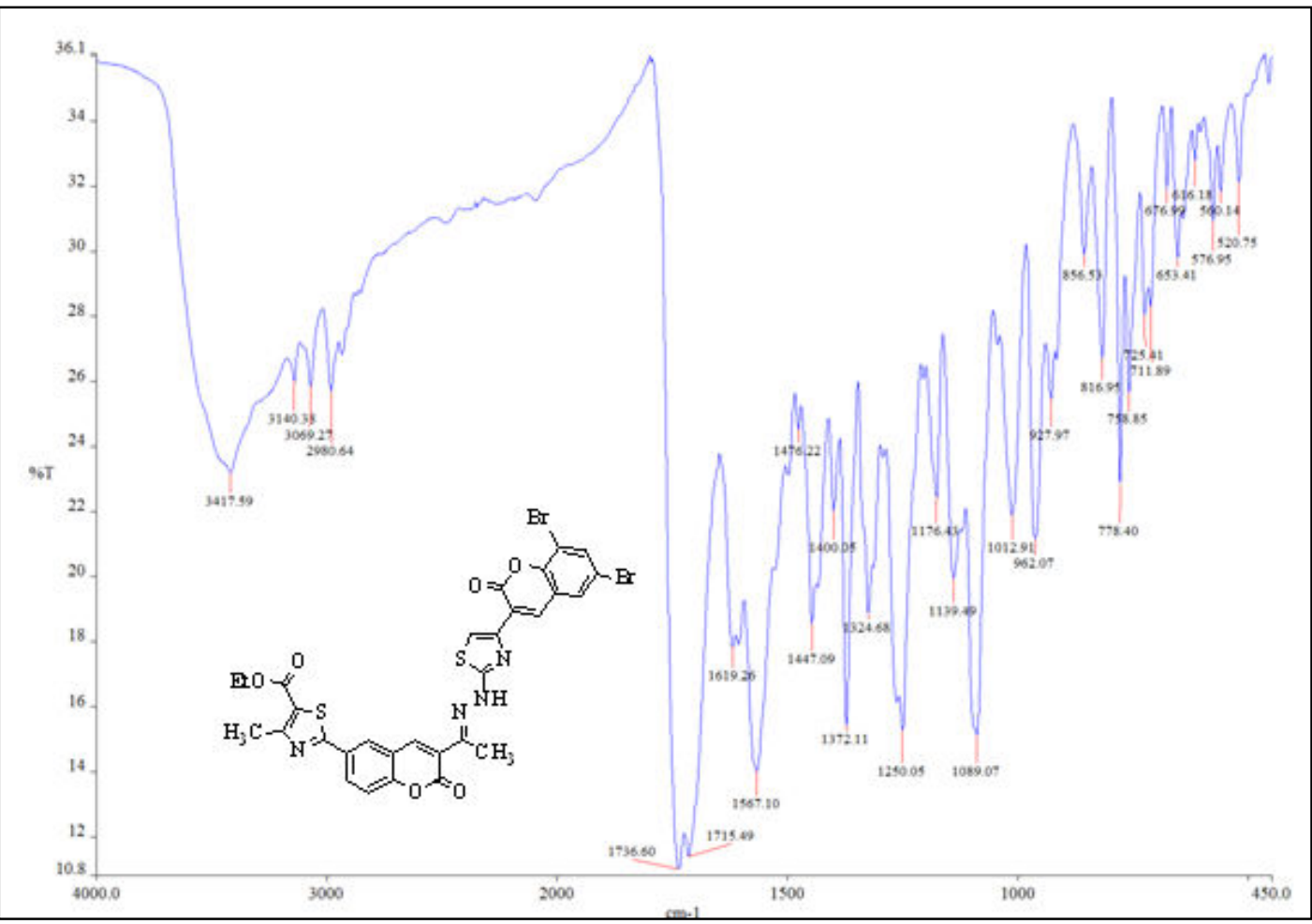




\section{IR (KBr) spectrum of compound 61}

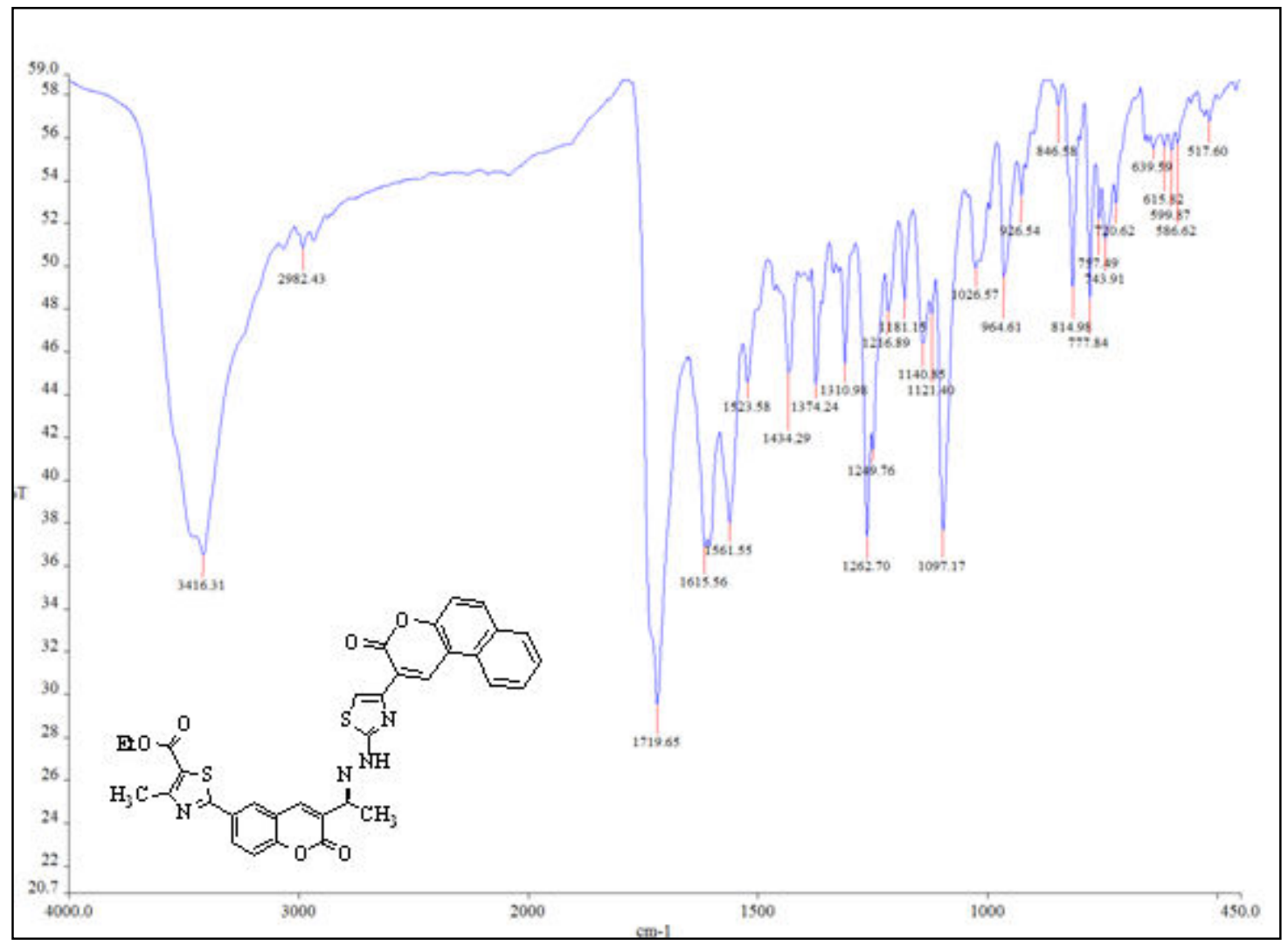

